\title{
Topological approach to quantum Hall effects and its important applications: higher Landau levels, graphene and its bilayer
}

\author{
Janusz Jacak, Patrycja Łydżba ${ }^{a}$, and Lucjan Jacak \\ Faculty of Fundamental Problems of Technology, Wroclaw University of Technology, Wyb. Wyspiańskiego 27, \\ 50-370 Wrocław, Poland
}

Received 25 January 2017 / Received in final form 16 March 2017

Published online 18 May 2017

(C) The Author(s) 2017. This article is published with open access at Springerlink.com

\begin{abstract}
In this paper the topological approach to quantum Hall effects is carefully described. Commensurability conditions together with proposed generators of a system braid group are employed to establish the fractional quantum Hall effect hierarchies of conventional semiconductors, monolayer and bilayer graphene structures. Obtained filling factors are compared with experimental data and a very good agreement is achieved. Preliminary constructions of ground-state wave functions in the lowest Landau level are put forward. Furthermore, this work explains why pyramids of fillings from higher bands are not counterparts of the well-known composite-fermion hierarchy - it provides with the cause for an intriguing robustness of $\nu=\frac{7}{3}, \frac{8}{3}$ and $\frac{5}{2}$ states (also in graphene). The argumentation why paired states can be developed in two-subband systems (wide quantum wells) only when the Fermi energy lies in the first Landau level is specified. Finally, the paper also clarifies how an additional surface in bilayer systems contributes to an observation of the fractional quantum Hall effect near half-filling, $\nu=\frac{1}{2}$.
\end{abstract}

\section{Introduction}

Although the first experiment allowing for an observation of the fractional quantum Hall effect (FQHE) was carried out by Tsui, Stormer and Gossard in 1983 [1], the real nature of this phenomenon is still not understood. What do we know for sure is that the FQHE is a collective peculiarity resulting from strong interactions between particles [2] and a specific two-dimensional (2D) topology. Additionally, one of the basic prerequisites for an appearance of Hall-like correlations is a flat band consisted of states with the same kinetic energy - as in the case of almost degenerated Landau levels (LLs) in the presence of interactions. However, recent experiments on suspended graphene samples [3] revealed that the high value of mobility - not considered earlier - also plays a triggering role. This surprising feature puts into question all theories, which are based entirely on particle interactions and neglect topology requirements, since they may not embrace the true physics of Hall systems. The well-known Jain's theory $[4,5]$ - which introduces the concept of composite fermions (CFs) - has its place among these models. Its popularity is owned to the fact that it can be used to explain most (but not all) of the fractions evidenced experimentally in the lowest Landau level (LLL). Thus, it was a great disappointment when scientists discovered that the FQHE hierarchies from higher Landau

\footnotetext{
${ }^{a}$ e-mail: patrycja.lydzba@pwr.edu.pl
}

levels $(n>0)$ are not counterparts of the Jain's hierarchy, which has been employed (by that time) with a great success [6-12]. Furthermore, the appearance of additional even-denominator states - like $\frac{5}{2}$ or $\frac{7}{2}$ - in transport measurements was impossible to predict within this local, quasi-particle model. What was especially confusing - in two-subband systems (wide quantum wells) - these paired states were not developing when the Fermi energy was placed in the zeroth Landau level of the higher-energy subband [12]. Their emergence required a partial filling of the first Landau level. What is more, the presence - in bilayer systems [13-15] - of incompressible $\nu=\frac{1}{2}$ was also confusing for Jain's theory supporters. For all of these reasons, in this paper, we introduce the cyclotron subgroups model [16-23], which is based on the topology of twodimensional systems upon strong magnetic fields, to the issue of the FQHE in higher Landau levels, in monolayer and bilayer graphene.

The article is divided as follows: the second section introduces the concept of a topological approach to quantum Hall effects (concerning especially on a point-by-point derivation of commensurability conditions and FQHE hierarchies in the LLL); the following section explains the difference between particle cyclotron orbit (as a representative of a braid group element) in the lowest and higher Landau levels and its most significant consequences (a possible single-loop FQHE, a particle pairing effect and other) - the obtained results are compared with experimental studies; the last section gives the prescription 
on how to use the cyclotron subgroup model in monolayer and bilayer graphene systems. The latter contains a topological explanation of the occurrence of $\nu=\frac{1}{2}$ incompressible state in bilayer materials. The comparison with experimental findings is also provided. At the end of the paper one can find the concluding remarks.

\section{Cyclotron subgroups model}

\subsection{Full braid group and quantum statistics}

The topological approach to quantum Hall effects - called the cyclotron subgroup model [16-18,20-23] - is entirely based on the mathematical concept of braid groups. The full braid group of a multi-particle system contains all closed trajectories encircled in an appropriate configuration space and organised in homotopy classes [24,25],

$$
\pi_{1}(\Omega)=\pi_{1}\left(\left(M^{N} \backslash \Delta\right) / S_{N}\right)
$$

where, $\pi_{1}(\Omega)$ - called the full braid group - is a homotopy group of a system configuration space $(\Omega), N$ stands for the number of electrons, $M$ is a manifold (we will consider only simply-connected) on which particles are placed, $S_{N}$ denotes the permutation group and $\Delta$ (subtracted to preserve the number of particles) removes points for which particle positions (at least two) are the same. Note that due to the indistinguishability of particles - initial and final orderings of carriers, while constructing elements of $\pi_{1}$, may differ by a permutation. It is also worth adding that a configuration space for more than one particle is always multiply connected, as a result of the $\Delta$ set ejection (while constructing configuration space we ensure that coordinates of a point from $M^{N}$ manifold, connected with positions of classical particles, do not overlap $-\Omega$ consists topological defects). Finally, the full braid group is generated with elements (braids) $\sigma_{i}$, which describe exchanges of neighbouring (classical, as $M^{N}$ coordinates) particles $i$ th and $(i+1)$ th.

Before we proceed to a detailed explanation of the cyclotron subgroup model, let us comment the appearance of paths in a $\pi_{1}$ definition. It is very important to understand that quantum particles do not travel braid trajectories, since (in the quantum world) particles do not travel in trajectories at all. However, in agreement with the general rules of quantization, a multi-particle wave function $\Psi_{N}$ needs to transform according to a one-dimensional unitary representation (1DUR) of the system braid group [26]. In other words, when classical particles (as arguments of $\Psi_{N}$ or $M^{N}$ coordinates) encircle a loop in their configuration space, then the wave function acquires a phase shift in consonance with the 1DUR of the braid, which corresponds to this closed path.

Moreover, one can achieve the matching of topological properties with quantum properties of a multi-particle system by a quantization according to the Feynman path integral method $[27,28]$,

$$
\begin{aligned}
& K(b, a)=\lim _{\delta t \rightarrow 0}\left(\frac{m}{2 \pi i \delta t}\right)^{n / 2} \\
& \times \int\left(\prod_{j=1}^{n-1} d q_{j}\right) e^{i \sum_{j=0}^{n-1}\left(\frac{m \dot{q}_{j}}{2}-V\left(q_{j}\right)\right) \delta t}=\int d \lambda e^{i S\left(\lambda_{b, a}\right)}
\end{aligned}
$$

where, $K$ is the propagator - the probability of transition from $a$-point at $t=t_{1}$ to $b$-point at $t=t_{2}, q_{0}, q_{1}, \ldots, q_{n}$ are midpoints of a chosen trajectory $\lambda_{a, b}, V$ stands for the potential, $m$ symbolizes the particle mass and $\delta t$ stands for a time increment. Additionally, $d \lambda$ is an interval in the trajectory space. For all non-simply connected configuration spaces a supplementary summation over classes of homotopic $a$-point to $b$-point trajectories - with weight factors determined by a 1DUR of the braid group [29] has to be implemented in the integral definition. If one considers two non-homotopic trajectories linking points $a$ and $b$, one will notice that they can be considered as identical open paths $\lambda_{a, b}$, but with different $\backslash$ non-equivalent closed loops attached to them. Since these trajectories fall into separate homotopy classes, thus a single measure $d \lambda$ in the path space cannot be defined. These collections can be, however, distinguished from each other with the use of elements from the system braid group, hence an additional summation over $\pi_{1}(\Omega)$ braids should be included in the path integral with appropriate unitary factors. Finally, the propagator takes the form of [29],

$$
K(b, a)=\sum_{l \in \pi_{1}} e^{i \alpha_{l}} \int d \lambda_{l} e^{i S\left(\lambda_{b, a}^{l}\right)}
$$

where, $l$ enumerates the braid group elements and $\lambda_{b, a}^{l}$ indicates an open trajectory $\lambda_{b, a}$ between $a$ and $b$ points with $l$ th loop added. It is worth to mention that different representations of the full braid group (and also different forms of $\pi_{1}$ ) give rise to distinct quantum statistics. For example, one can obtain bosons, $\sigma_{i}=e^{i 0}$, but also fermions, $\sigma_{i}=e^{i \pi}$, using 1DURs of the permutation group $S_{N}$. If the full braid group has a far more rich structure - like in the case of 2D manifolds - the analyzation of possible 1DURs may lead to an infinite number of quantum particles (including bosons and fermions) with fractional statistics, $e^{i \theta}$ with $\theta \in[0,2 \pi)[16,17,20-22]$. The latter are called anyons.

In the standard, Jain's [4,5], model of the fractional quantum Hall effect it is impossible to distinguish composite from ordinary fermions, because they both experience the same quantum statistics $[16,20]$. This is due to the fact that 1DURs are periodic with a periodicity of $2 \pi$ and so $e^{i p \pi}=e^{i \pi}$. In order to deal with this issue, we suggest to associate CFs with the appropriately constructed braid subgroups of $\pi_{1}$ and in this way to differentiate them from ordinary fermions characterized with the full braid group. 


\subsection{System braid group and strong magnetic field}

Note that some external factors may change the form of braid group describing the system, by - for instance preventing the realization of some types of (classes of homotopical) trajectories [16-23]. This effect (the reshaping of a group) occurs in two-dimensional manifolds after the inclusion of a strong magnetic field - when a classical cyclotron motion confines the variety of accessible braids. In other words, if a particle cyclotron orbit (as a representative in the $M$ space of a braid group element from the $\Omega$ space) is too small to reach a neighbouring carrier, then the occurrence of single-loop exchanges (representing generators $\sigma_{i}$ of $\pi_{1}$ ) is unallowed. This is the case of the LLL fractional filling.

It is worth to mention, the multi-particle system needs to have a determined statistics to form a collective Halllike state. Since the FQHE is actually observed, thus the exchanges (of some type) have to be restored and the particle cyclotron orbit (representing a braid group element) needs to be enhanced, somehow. In a theory this can be achieved in various ways, e.g., by screening the external magnetic field - like for electrons with pinned flux quanta $[4,5]$, or by diluting a local charge - as for electrons with $q$-fold vortices [4]. However, a natural way to accomplish this enhancement is to exclude inaccessible homotopy classes from the braid group describing the system. If - for an arbitrary filling factor - the remaining braids can be organised in a subgroup of $\pi_{1}$, then the fractional quantum Hall state can be formed. Additionally, the generators $b_{i}$ of this so-called cyclotron subgroup represent new exchanges (a closed path of any particle, which represents $b_{i} b_{i}$ element, is characterized with a sufficiently large area to reach one of classical-carrier neighbours). Finally, it should be clear that when a $2 \mathrm{D}$ system is placed in a strong magnetic field, then the summation in a definition of the Feynman propagator has to be confined to elements of this restricted (not full) braid group.

\subsection{Cyclotron subgroups and basic hierarchy of FQHE}

Cyclotron subgroups do not contain simple \loopless exchanges of classical carriers (representing $\sigma_{i}$ ), but they are consisted solely of elements belonging to the full braid group (to have a $\pi_{1}$ subgroup character) which are still accessible after a magnetic field is applied. Thus, they are generated with multi-loop exchange braids [16,17,20-22],

$$
b_{i}^{(p)}=\left(\sigma_{i}\right)^{p}, \quad p=3,5,7, \ldots, \quad i=1,2, \ldots N-1,
$$

where $p$ enumerates different types of cyclotron subgroups. It is important to point up that generators can have more complicated form - they can comprise various $\sigma_{i}, \sigma_{j}, \ldots, \sigma_{k}$ elements (for $i \neq j \neq \ldots \neq k$ ). The latter case will be described further in the paper. Note that the $b_{i}^{(p)}$ elements are represented by exchanges of neighbouring, $i$ th and $(i+1)$ th, classical particles with additional $\frac{p-1}{2}$ loops. However, all exchanges have to form open trajectories and, thus, only entire loops can be added to the representative of a $\sigma_{i}$ generator. In other words, the number of supplementary loops must be an integer, what enforces an odd value of $p$. Already at this point one can suspect that this stands for a straightforward and topological explanation, why the fractional quantum Hall effect is observed only for odd denominator fractions (as a first approximation, ignoring other effects like a pairing of particles). It should be also emphasized that the $b_{i}^{(p)}$ element is not represented by a path consisted of $p$ simple exchanges of electrons, since they cannot be performed in a presence of a strong magnetic field in $2 D$ spaces. The (multi-loop) exchange is completed only after a realization of the total trajectory defined by the cyclotron subgroup generator. Furthermore, a cyclotron trajectory is always a closed path and its simplest version is obtained with the use of two multi-loop exchanges (when a statistics is determined and a system is described with a certain subgroup). Finally, the cyclotron trajectory (as a mapping of a braid group element from $\Omega$ on a $M$ manifold) has $p$ loops - this quantity is equal to the total number of external magnetic field flux quanta attributed to a particle in the case of $\frac{1}{p}$ filling of the LLL.

The one-dimensional unitary representations of a cyclotron subgroup have the same form as 1DURs of $\pi_{1}$ restricted to this subgroup [16,17,20-22],

$$
b_{i}^{(p)} \rightarrow e^{i p \alpha}, \quad p=3,5,7, \ldots, \quad i=1,2, \ldots N-1,
$$

where $\alpha \in[0,2 \pi)$. In a topological approach to quantum Hall effects we argue that different pairs of parameters $(\alpha, p)$ describe distinct composite anyons (for $\alpha=$ $\pi$ - composite fermions), connected rather with original anyons in the case of lack of an external magnetic field (for $\alpha=\pi$ - original fermions). As it was already mentioned, the multi-particle wave function $\Psi_{N}$ transforms according to the appropriate 1DUR of a braid group describing the system (e.g., a cyclotron subgroup). Hence, when two composite anyons are exchanging positions (as arguments of $\Psi_{N}$ ), the multi-particle wave function acquire a phase shift equal to $p \alpha$ (each supplementary loop appearing in the cyclotron subgroup generator definition results in an additional phase gain of $\left.2 \alpha ; \Delta \beta=\alpha+2 \alpha \cdot \frac{p-1}{2}=p \alpha\right)$. Finally, $\Delta \beta=p \pi$ for composite fermions and it is equivalent to the phase change picked up by the Laughlin function (LF) [2],

$$
\Psi_{N}^{(L)}\left(z_{1}, z_{2}, \ldots, z_{N}\right)=\prod_{i, j=1, i>j}^{N}\left(z_{i}-z_{j}\right)^{p} e^{-\sum_{i=1}^{N} \frac{\left|z_{i}\right|^{2}}{4 l^{2}}}
$$

where, $z_{j}=x_{j}+i y_{j}$ is a complex particle position and $l=\sqrt{\frac{\hbar c}{e B}}$ is a magnetic length (distance). As one can see, the composite fermions are not complexes of electrons with external magnetic field flux quanta pinned to them (which recover Laughlin correlations using Aharonov-Bohm effect [30]), but are rightful quantum particles described with cyclotron subgroups of $\pi_{1}$. Note that CFs cannot be mixed up with ordinary fermions just as fermions cannot be mixed up with bosons. Thus, while performing exact 
diagonalisations, not all antisymmetrical wave functions but only those, which transform itself according to the 1DUR of the proper cyclotron subgroup - should be taken into account.

Let us now demonstrate the effective cyclotron path enlargement process in less mathematical and more illustrative way. It is out of a question that, in the presence of an external magnetic field, trajectories representing elements of a system braid group are of cyclotron orbit type even when Coulomb interactions are considered (though they are not simple circles then). The surface they encircle cannot be, however, determined with the use of a classical free-particle radius, $R_{c}=\frac{m v}{e B}$. Instead, the cyclotron orbit is defined by the archetype of correlated incompressible state - the IQHE state. For this reason, the plaque with the area,

$$
\frac{S}{N_{0}}=\frac{h c}{e B}
$$

which embraces exactly one flux quantum $\left(N_{0}\right.$ stands for a level degeneracy and a number of flux quanta), is the definition of the cyclotron orbit (as a mapping of a braid group element) in the whole LLL. Let us emphasise that the cyclotron path (as a braid group element) defined in this manner fits perfectly to the interparticle distance $\left(\frac{S}{N_{0}}=\frac{S}{N}\right)$ and consists of two simple $\backslash$ loopless exchanges of neighbouring particles (as $M^{N}$ coordinates or arguments of a wave function) $[20,22]$. Note that for degenerated Landau levels all particles should encircle the same cyclotron area, even though the velocity cannot be properly determined (its coordinates do not commute). This is due to the fact that all states have identical kinetic energy and it is reasonable to assume that the classical particles are also characterized with identical cyclotron surface (similarly as in the semiclassical Onsager-Lifshitz approach).

Lets consider a situation created after raising of an external magnetic field strength. The number of particles $(N)$ is now reduced comparing to the level degeneracy $\left(N_{0}\right)$. Additionally, there is more than one flux quantum attributed to a single classical carrier. For example, $\frac{B S}{N} / \frac{h c}{e}=p$ for $\frac{N}{N_{0}}=\frac{1}{p}$ filling. In spite of this, the surface of a cyclotron orbit still embraces one $\phi_{0}=\frac{h c}{e}-$ it is too small to reach a neighbouring carrier $\left(\frac{S}{N_{0}}<\frac{S}{N}\right)$ and it cannot be implemented as a double simple $\backslash$ loopless exchange. Since the FQHE is widely observed in experiments, braid-group exchanges of particles have to be possible, even though generators of the full braid group cannot be defined. We argue that (for some fractions) multi-loop trajectories can have sufficient dimensions to reach neighbouring particles and allow for multi-looped exchanges in the system (representing cyclotron subgroup generators). In order to prove this point consider a path consisted of $p$ loops (bear in mind that it can be generated as $b_{i}^{(p)} b_{i}^{(p)}$ element, if and only if corresponding $b_{i}^{(p)}$ exchanges can be defined). In $3 \mathrm{D}$ spaces each loop of a $p$-looped trajectory adds a new portion of field flux quanta piercing the path, in the same manner as circumvolution adds a new surface

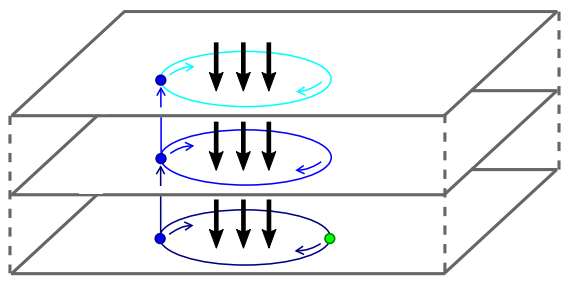

a) $3 \mathrm{D}$

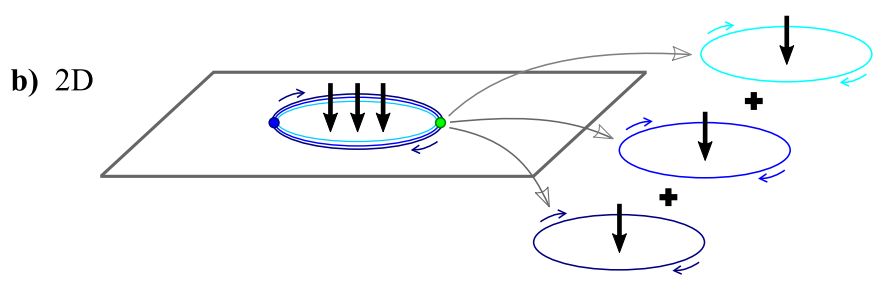

Fig. 1. (a) In $3 \mathrm{D}$ spaces each loop of a multi-looped (here: $p=3$ ) cyclotron path adds a new portion of field flux quanta piercing this trajectory, in the same manner as circumvolution adds a new surface to a coil. (b) In the same time, in 2D manifolds a loop cannot enhance the surface encircled by the trajectory and therefore cannot enhance its embraced flux. Finally, all three loops must share the same total quantity $\frac{B S}{N}$, which passes through a single-looped trajectory. Thus, an arbitrary loop embraces a diminished portion of a magnetic field flux, which appears to be equal to one in the case of $\nu=\frac{1}{3}$ filling $\left(\frac{3 \cdot \phi_{0}}{3}=\phi_{0}=\frac{h c}{e}\right)$; the same quantity as for the cyclotron orbit in the LLL).

to a coil. In opposition, in 2D manifolds a loop cannot enhance the surface encircled by the trajectory and therefore cannot enhance its embraced flux $[16,17,20-22]$. Finally, all $p$ loops must share the total, per particle quantity, $\frac{B S}{N}$, embraced by a single-looped path (Fig. 1). Thus, an arbitrary loop embraces a diminished portion of a magnetic field flux per particle, which is equal to one for $\nu=\frac{1}{p}$ filling $\left(\frac{p \phi_{0}}{p}=\phi_{0}=\frac{h c}{e}\right)$; the same quantity as for the cyclotron orbit in the LLL). Finally (when the LLL is $1 / p$ filled), the $p$-looped paths encircle grater surface than single-looped ones, what leads to the effective enhancement of a cyclotron orbit (as a mapping on $M^{N}$ space of a trajectory performed in a configuration space) and allows for the occurrence of (multi-looped, defined by $b_{i}^{(p)}$ ) exchanges in the system.

The reasoning described above is sufficient for understanding, why the FQHE occurs most robustly for filling factors $-\nu=\frac{1}{p}(p-$ odd $)-$ which belong to the so-called basic set of the LLL. In the next subsection we will demonstrate that particles may experience the Hall effect also for some other, properly selected, fillings. Additionally, the topological approach allows for solving of the the odd-denominator puzzle, simply by noticing that any cyclotron (multi-looped) trajectory divided into half must produce a proper exchange (open) trajectory so $p$ needs to be an odd number. If one takes into account this two results it becomes obvious, the most robust fractional Hall-like state is related to the simplest multilooped exchange with only one additional loop $\left(b_{i}^{(3)}\right)-$ $\frac{p-1}{2}=1 \leftrightarrow p=3$ - hence it created for a $1 / 3$ filling 
of the LLL. It is also worth to emphasize that the mathematically non-trivial cyclotron subgroups model leads to surprisingly compact commensurability conditions, which allow for the determination of the FQHE filling factors. The main meaning of mentioned conditions can be described in one sentence: the quantum Hall effect can be realized in the system if and only if the surface encircled by a (single- or multi-looped) cyclotron path is equivalent to the area attributed to a single particle. Thus, in the LLL, this leads to an expression [18,19,22,23],

$$
p \frac{h c}{e B}=p \frac{S}{N_{0}}=\frac{S}{N} \text {. }
$$

Finally, one arrives with the expected solution, $\nu=\frac{N}{N_{0}}=$ $\frac{1}{p}(p-$ odd $)$. The generalization of commensurability conditions to higher Landau levels requires a bit of commenting. Firstly, it should be obvious that cyclotron orbit dimensions need to be proportional to the bare (without a crystal field) kinetic energy, hence the cyclotron surface is quickly increasing $-\propto(2 n+1)$ - with the Landau level index, $n$. Secondly, in higher Landau levels, due to this rapid growth, exist the possibility that the cyclotron trajectory has too large dimensions to fit the interparticle separation. In this situation, the area encircled by a cyclotron path also must be enhanced to allow for the exchanges of particles with their higher-order neighbours. Conclusively, the hierarchy of fillings for the FQHE can be determined from the following equation [22],

$$
\begin{aligned}
p(2 n+1) \frac{h c}{e B} & =p(2 n+1) \frac{S}{N_{0}}=\frac{a S}{N-\gamma N_{0}} \rightarrow \nu=\frac{N}{N_{0}} \\
& =\gamma+\frac{a}{(2 n+1) p}
\end{aligned}
$$

where, integer $\gamma$ is the number of completely filled Landau sublevels and integer $a$ reflects the complexity of accessible exchange braids.

At the end of these considerations, we would like to point out that Coulomb repulsion forces play a very important role also in the topological approach to quantum Hall effects $[16,17,22,23]$. As one could already notice, interactions rigorously protect the minimal surface attributed to a single particle, $\frac{S}{N}$, and they do not let neighbouring carriers (classical - as arguments of $\Psi_{N}$ ) to violate this guarded area. The formulation of a cyclotron subgroup model for non-interacting systems would be precluded, since along the trajectories belonging to $\pi_{1}$ some other paths could - sometimes - appear too (could be allowed for a system).

\subsection{FQHE hierarchy out of $\frac{1}{q}$ fillings}

We would like to point up that - in the case of $\frac{1}{p}$ filling factors - the last loop of a $p$-looped trajectory experiences a lower magnetic field (a reduced portion of flux quanta), just like composite fermions in the standard model $[4,5]$.
Thus, CFs are not complexes of electrons with $p-1$ localized flux tubes but they are rightful quantum particles, just like fermions or bosons, with their statistic determined from 1DURs of different cyclotron subgroups. It should be noticed that - in the topological approach the equivalence between the number of magnetic field flux quanta attributed to a single particle in a system and the number of loops in a cyclotron path (representing a braid group element) holds only for the basic set of fillings, what was described in details in a previous subsection. Out of $\nu=\frac{1}{p}$ this correspondence cannot be assumed, since the number of flux quanta per particle becomes fractional. In the model of composite fermions one deals with a conception that, even for filling factors beyond the basic set, the amount $(p-1)$ of quanta pinned to particles is integer and satisfies the condition,

$$
\pm \frac{1}{m} \phi_{0}=\nu^{-1} \phi_{0}+(p-1) \phi_{0} .
$$

In other words, the FQHE can be realized in the system if and only if CFs completely fill $m=1,2,3, \ldots$ Landau levels in a diminished effective magnetic field. This leads to the well-known Jain's hierarchy $[4,5]$,

$$
\nu=\left((p-1) \pm \frac{1}{m}\right)^{-1}=\frac{m}{m(p-1) \pm 1} .
$$

It's rather understandable that fluxes attached to electrons are only artificial objects and do not exist. Considering, however, a great success of the CF model one can conclude that this pinned quanta provide a convenient, though simplistic, model of additional loops belonging to a cyclotron trajectory $[16,17,20,21]$. In order to match these two approaches one can assume that all $p-1$ loops of a multi-looped path (as mappings of $b_{i} b_{i}$ elements) embrace a complete flux quantum per particle (each), while the last one takes away the remaining portion per particle fractional for $\nu \neq \frac{1}{p}$.

At the end of this section let us note that a cyclotron orbit in the LLL is still defined with one $\phi_{0}$ and the quantization rule is not obeyed with regard to the last loop of a multi-looped path (for $\nu \neq \frac{1}{p}$ ). However, when the embraced portion is equal to the $m$ th part of a flux quantum, $\frac{h c}{m e B}$, one can suspect that the organization of a collective correlation can be achieved. In this situation the last loop fits to the separation of an arbitrary particle (as a $M^{N}$ coordinate) and its $m$ th neighbour, and allows those classical carriers to exchange $\left(m \frac{h c}{m e B}=\frac{h c}{e B}=\phi_{0}\right)$. Additionally, a sign of the residual magnetic field flux can be - obviously positive, but also negative. The latter case is expected to be associated with an eight-shape multi-looped trajectory, whose last loop is rolled in an opposite direction to one forced by the external magnetic field orientation.

Ultimately, the generalized (out of the most robust set) hierarchy can also be established for higher Landau levels [22],

$$
\nu=\gamma+\frac{1}{(2 n+1)\left(\frac{p-1}{a} \pm \frac{1}{m}\right)}=\gamma+\frac{m}{(2 n+1)\left(m \frac{p-1}{a} \pm 1\right)}
$$


where, $m$ and $a$ are integers which satisfy the following condition, $a \bmod m=0$, and $\gamma$ stands for the number of completely filled Landau sublevels. This means that cyclotron subgroups are generated by,

$$
\begin{aligned}
b_{i}^{(p), \pm}= & \left(\sigma_{i} \sigma_{i+1} \ldots \sigma_{i+a-1} \ldots \sigma_{i+1}{ }^{-1} \sigma_{i}^{-1}\right)^{p-1} \\
& \times \sigma_{i} \sigma_{i+1} \ldots \sigma_{i+m-1}{ }^{ \pm 1} \ldots \sigma_{i+1}{ }^{-1}{\sigma_{i}}^{-1}
\end{aligned}
$$

where, plus sign (also in Eq. (12)) stands for the generators represented by paths with the last loop encircled in a direction forced by the external magnetic field, whereas minus - by paths with the last loop encircled in an opposite direction to one forced by the $B$ field.

Presented considerations seem to be sufficient to conclude on a preliminary construction of a ground-state wave function in the LLL (we have currently investigated only a hierarchy with a plus sign in a denominator),

$$
\Psi_{N}=\prod_{i<j}\left(z_{i}-z_{j}\right)^{p} \prod_{k<l}\left(z_{k}-z_{l}\right)^{p-1} e^{-\frac{1}{4 l_{0} 2} \sum_{i}\left|z_{i}\right|^{2}}
$$

where,

$$
\begin{aligned}
& |k-l| \bmod a=0,|k-l| \bmod m \neq 0, \\
& |i-j| \bmod m=0
\end{aligned}
$$

We point out that the above proposal takes into account the order of accessible exchanges (the order of neighbours which can be connected by a series of accessible exchanges) and reflects the complexity of a generator. Additionally, we do not need to implement any projection operators to obtain a holomorphic function entirely located in the LLL.

\section{Commensurability conditions and higher Landau levels}

In this part of the paper we are going to use the commensurability conditions (introduced earlier) to derive all filling factors - from higher Landau levels - for which a 2DEG system can form collective Hall-like states. As we already mentioned, the area of a particle cyclotron orbit (classical - as a representative of a system braid group element) rapidly increases $(\propto 2 n+1)$ with the Landau levels index $n$. Thus, in higher bands there exists more promising - that may lead to the ordinary or single-loop FQHE $[16,17,23]$ - situations, all of which are listed below:

1. Firstly, the cyclotron orbit (without any additional loops - as a mapping of $\sigma_{i} \sigma_{i}$ ) may fit perfectly to the interparticle separation. For particles located on the first Landau level this leads to expressions,

$$
\left\{\begin{aligned}
\frac{(2 \cdot 1+1) \cdot h c}{e B}=\frac{3 \cdot S}{N_{0}}=\frac{S}{N-2 N_{0}} \rightarrow & \nu=\frac{N}{N_{0}}=\frac{7}{3}, \\
& \text { up-spin subband } \\
\frac{(2 \cdot 1+1) \cdot h c}{e B}=\frac{3 \cdot S}{N_{0}}=\frac{S}{N-3 N_{0}} \rightarrow & \nu=\frac{N}{N_{0}}=\frac{10}{3}, \\
& \text { down-spin subband. }
\end{aligned}\right.
$$

A generalized condition for other Landau bands,

$$
\frac{(2 n+1) h c}{e B}=\frac{(2 n+1) S}{N_{0}}=\frac{S}{N-\gamma N_{0}} \rightarrow \nu=\gamma+\frac{1}{2 n+1}
$$

where, $\gamma=2,3,4, \ldots$ is the number of all completely filled spin sublevels. Note that all filling factors derived above are fractional and, thus, the quantum Hall effect is realized even though a multi-particle system is described with the full braid group and not one of its cyclotron subgroups. For this reason, this collective states correspond to the single-loop FQHE, which rather resembles the IQHE than ordinary fractional quantum Hall effect $[18,19,23]$. One should remark that this novel phenomenon can appear only for $n>0$ Landau levels. Finally, the Laughlin correlations - while considering the single-loop FQHE - are described with a $p=1$ power in the Jastrow polynomial and loopless exchanges (just like in the IQHE case), despite fractional quantization of a transverse resistivity (just like in the ordinary FQHE case).

2. In the first Landau level there are two additional possibilities, which lead to the quantum Hall effect formation in a system described with simple exchanges, $\sigma_{i}$. For example, the cyclotron orbit (as a representative of a braid group element) may fit perfectly to the distance between any arbitrary particle and its second neighbours (the single-loop FQHE),

$$
\left\{\begin{aligned}
& \frac{3 h c}{e B}=\frac{3 S}{N_{0}}=\frac{2 S}{N-2 N_{0}} \rightarrow \nu= \frac{N}{N_{0}}=\frac{8}{3}, \\
& \text { up-spin subband } \\
& \frac{3 h c}{e B}=\frac{3 S}{N_{0}}=\frac{2 S}{N-3 N_{0}} \rightarrow \nu= \frac{N}{N_{0}}=\frac{11}{3}, \\
& \text { down-spin subband. }
\end{aligned}\right.
$$

The cyclotron orbit may, also, have sufficient surface to allow exchanges of electrons (as coordinates of a point in $M^{N}$ space) with their third neighbours,

$$
\left\{\begin{aligned}
\frac{3 h c}{e B}=\frac{3 S}{N_{0}}=\frac{3 S}{N-2 N_{0}} \rightarrow \nu= & \frac{N}{N_{0}}=3, \\
& \text { up-spin subband } \\
\frac{3 h c}{e B}=\frac{3 S}{N_{0}}=\frac{3 S}{N-3 N_{0}} \rightarrow \nu=\frac{N}{N_{0}}=4, & \text { down-spin subband. }
\end{aligned}\right.
$$

In this situation carriers (as $\Psi_{N}$ arguments) are experiencing the integer quantum Hall effect in completely filled subbands of the first Landau level. This agrees with a simple intuition: since the IQHE in the LLL is realized when adjoining particles can exchange, thus in the first Landau level - where the classical cyclotron orbit is three times larger - the IQHE is realized when a carrier can exchange with its third neighbour [18,19,21-23].

A generalized condition for other Landau bands,

$$
\frac{(2 n+1) h c}{e B}=\frac{(2 n+1) S}{N_{0}}=\frac{a S}{N-\gamma N_{0}} \rightarrow \nu=\gamma+\frac{a}{2 n+1}
$$


Table 1. Typical semiconductors: filling factors obtained from commensurability conditions for all spin branches of the first Landau level. FQHEs stands for single-loop fractional Hall states. A few hole states have been placed in brackets. Fractions observed experimentally are marked with pink colour [6-10,31].

\begin{tabular}{clll}
\hline LL subb. & IQHE, FQHEs & Hall metal & FQHE \\
\hline $1 \uparrow$ & $\frac{13}{6}, \frac{25}{12}, \ldots$ & $2+\frac{1}{3\left(2-\frac{1}{m}\right)}$ & $\frac{7}{3}, \frac{20}{9}\left(\frac{25}{9}\right), \frac{11}{5}, \frac{46}{21}, \frac{59}{21}, \ldots$ \\
& $2+\frac{1}{3\left(2+\frac{1}{m}\right)}$ & $\frac{19}{9}, \frac{32}{15}, \frac{15}{7}, \frac{58}{27}, \frac{71}{31}, \ldots$ \\
& $2+\frac{1}{3\left(2 \cdot \frac{1}{2}-\frac{1}{m}\right)}$ & $\frac{8}{3}, \frac{22}{9}, \frac{12}{5}, \frac{50}{21}, \frac{64}{27}, \ldots$ \\
& $2+\frac{1}{3\left(2 \cdot \frac{1}{2}+\frac{1}{m}\right)}$ & $\frac{20}{9}\left(\frac{25}{9}\right), \frac{34}{15}, \frac{16}{7}\left(\frac{19}{7}\right), \frac{62}{27}, \frac{76}{33}, \ldots$ \\
& $2+\frac{1}{3\left(2 \cdot \frac{1}{3}-\frac{1}{m}\right)}$ & $3, \frac{8}{3}, \frac{13}{5}, \frac{18}{7}\left(\frac{17}{7}\right), \frac{23}{9}, \frac{28}{11}, \frac{33}{13}\left(\frac{32}{13}\right), \ldots$ \\
\hline $1 \downarrow$ & $3+\frac{1}{3\left(2-\frac{1}{m}\right)}$ & $\frac{10}{3}\left(\frac{11}{3}\right), \frac{29}{9}, \frac{16}{5}\left(\frac{19}{5}\right), \frac{67}{21}, \frac{86}{27}, \ldots$ \\
& & $3+\frac{1}{3\left(2+\frac{1}{m}\right)}$ & $\frac{28}{9}, \frac{47}{15}, \frac{22}{7}, \frac{85}{27}, \frac{104}{33}, \ldots$ \\
& & $3+\frac{1}{3\left(2 \cdot \frac{1}{2}-\frac{1}{m}\right)}$ & $\frac{11}{3}\left(\frac{10}{3}\right), \frac{31}{9}, \frac{17}{5}\left(\frac{18}{5}\right), \frac{71}{21}, \frac{91}{27}, \ldots$ \\
& & $3+\frac{1}{3\left(2 \cdot \frac{1}{2}+\frac{1}{m}\right)}$ & $\frac{29}{9}, \frac{49}{15}, \frac{23}{7}, \frac{89}{27}, \frac{109}{33}, \ldots$ \\
& & $3+\frac{1}{3\left(2 \cdot \frac{1}{3}-\frac{1}{m}\right)}$ & $4, \frac{11}{3}\left(\frac{10}{3}\right), \frac{18}{5}\left(\frac{17}{5}\right), \frac{25}{7}, \ldots$ \\
\hline
\end{tabular}

where $a$ is an integer number and determines which neighbours can exchange (in a system described by corresponding filling factor).

3. It is also possible that an electron (single-looped) cyclotron orbit is too small to allow for the exchanges of neighbouring particles (of $M^{N}$ coordinates). In this case, one needs to investigate whether the multi-looped trajectories have sufficient area to match the interparticle distance and to admit the existence of multilooped exchanges $-b_{i}^{(p)}$ with odd $p$. We have already devoted a lot of time and effort to explain this issue in the previous section, thus we are going to present only final results here.

The commensurability conditions in the first Landau level (Tabs. 1 and 2),

$$
\left\{\begin{aligned}
p \frac{3 h c}{e B}=p \frac{3 S}{N_{0}}=\frac{a S}{N-2 N_{0}} \rightarrow \nu= & \frac{N}{N_{0}}=2+\frac{a}{3 p}, \\
& \text { up-spin subband } \\
p \frac{3 h c}{e B}=p \frac{3 S}{N_{0}}=\frac{a S}{N-3 N_{0}} \rightarrow \nu= & \frac{N}{N_{0}}=3+\frac{a}{3 p}, \\
& \text { down-spin subband. }
\end{aligned}\right.
$$

One can also establish the generalized (Jain-like) hierarchies,

$$
\left\{\begin{array}{lc}
\nu=2+\frac{1}{3\left(\frac{p-1}{a} \pm \frac{1}{m}\right)}, & \text { up-spin subband } \\
\nu=3+\frac{1}{3\left(\frac{p-1}{a} \pm \frac{1}{m}\right)}, & \text { down-spin subband }
\end{array}\right.
$$

where $p$ is an odd number of loops in a multi-looped cyclotron path (as a mapping of a trajectory performed in a configuration space) and integer $m$ stands for a magnetic field flux portion per particle, which falls on the last loop.
Finally, in higher Landau bands,

$$
\begin{aligned}
p \frac{(2 n+1) h c}{e B} & =p \frac{(2 n+1) S}{N_{0}}=\frac{a S}{N-\gamma N_{0}} \\
& \rightarrow \nu=\gamma+\frac{a}{(2 n+1) p}
\end{aligned}
$$

and the generalized hierarchy equals,

$$
\nu=\gamma+\frac{1}{(2 n+1)\left(\frac{p-1}{a} \pm \frac{1}{m}\right)}
$$

where, integer $m$ stands for a magnetic field flux portion which falls on the last loop of a $p$-looped trajectory (and it defines which classical particles can exchange). Commensurability conditions constructed for $p-1$ loops experiencing different values of magnetic field flux per particle seem also reasonable to investigate. These loops can be divided into separate groups each collection comprises an even number of components, $\alpha_{i}$, and is determined by the effective fraction of a flux quantum attributed to a single particle, $\frac{1}{a_{i}} \phi_{0}$. In this case the cyclotron subgroups generators have the following form:

$$
\begin{aligned}
b_{i}{ }^{(p)}= & \left(\sigma_{i} \sigma_{i+1} \ldots \sigma_{i+a_{1}-1} \ldots \sigma_{i}{ }^{-1} \sigma_{i+1}{ }^{-1}\right)^{\alpha_{1}} \\
& \times\left(\sigma_{i} \sigma_{i+1} \ldots \sigma_{i+a_{2}-1}{ }^{ \pm 1} \ldots \sigma_{i}^{-1} \sigma_{i+1}{ }^{-1}\right)^{\alpha_{2}} \ldots
\end{aligned}
$$

while the pyramid of fillings is not a counterpart of a well-known CF hierarchy,

$$
\nu=y+\frac{1}{(2 n+1)\left(\alpha_{1} \frac{1}{a_{1}} \pm \alpha_{2} \frac{1}{a_{2}} \pm \ldots \pm \frac{1}{m}\right)}
$$


Table 2. Typical semiconductors: filling factors obtained from commensurability conditions for all spin branches of the second Landau level. IQHEp stands for the paired states, while FQHEs - for single-loop states. A few hole states have been placed in brackets. Fractions observed experimentally are marked with pink colour [6-10,31].

\begin{tabular}{cllllll}
\hline LL subb. & IQHE, FQHEs & Hall metal & & FQHE \\
\hline $2 \uparrow$ & $5, \frac{21}{5}, \frac{22}{5}, \frac{23}{5}, \frac{24}{5}$, & $\frac{41}{10}, \ldots$ & $4+\frac{1}{5\left(2-\frac{1}{m}\right)}$ & $\frac{21}{5}, \frac{62}{15}, \frac{103}{25}, \ldots$ & $4+\frac{1}{5\left(2+\frac{1}{m}\right)}$ & $\frac{61}{15}, \frac{102}{25}, \frac{143}{35}, \ldots$ \\
& & $4+\frac{1}{5\left(2 \cdot \frac{1}{2}-\frac{1}{m}\right)}$ & $\frac{22}{5}, \frac{64}{15}, \frac{106}{25}, \ldots$ & $4+\frac{1}{5\left(2 \cdot \frac{1}{2}+\frac{1}{m}\right)}$ & $\frac{62}{15}, \frac{104}{25}, \frac{106}{25}, \ldots$ \\
& & $4+\frac{1}{5\left(2 \cdot \frac{1}{3}-\frac{1}{m}\right)}$ & $\frac{23}{5}, \frac{22}{5}, \frac{109}{25}, \ldots$ & $4+\frac{1}{5\left(2 \cdot \frac{1}{3}+\frac{1}{m}\right)}$ & $\frac{21}{5}, \frac{106}{25}, \frac{149}{35}, \ldots$ \\
& & $4+\frac{1}{5\left(2 \cdot \frac{1}{4}-\frac{1}{m}\right)}$ & $\frac{24}{5}, \frac{68}{15}, \frac{112}{25}, \ldots$ & $4+\frac{1}{5\left(2 \cdot \frac{1}{4}+\frac{1}{m}\right)}$ & $\frac{64}{15}, \frac{108}{25}, \frac{152}{35}, \ldots$ \\
& & $4+\frac{1}{5\left(2 \cdot \frac{1}{5}-\frac{1}{m}\right)}$ & $5, \frac{14}{3}, \frac{24}{5}, \ldots$ & $4+\frac{1}{5\left(2 \cdot \frac{1}{5}+\frac{1}{m}\right)}$ & $\frac{13}{3}, \frac{22}{5}, \frac{31}{7}, \ldots$ \\
\hline $2 \downarrow$ & $5+\frac{1}{5\left(2-\frac{1}{m}\right)}$ & $\frac{26}{5}, \frac{77}{15}, \frac{128}{25}, \ldots$ & $5+\frac{1}{5\left(2+\frac{1}{m}\right)}$ & $\frac{76}{15}, \frac{127}{25}, \frac{178}{35}, \ldots$ \\
& & $5+\frac{1}{5\left(2 \cdot \frac{1}{2}-\frac{1}{m}\right)}$ & $\frac{27}{5}, \frac{79}{15}, \ldots$ & $5+\frac{1}{5\left(2 \cdot \frac{1}{2}+\frac{1}{m}\right)}$ & $\frac{77}{15}, \frac{129}{25}, \ldots$ \\
& & $5+\frac{1}{5\left(2 \cdot \frac{1}{3}-\frac{1}{m}\right)}$ & $\frac{28}{5}, \frac{27}{5}, \ldots$ & $5+\frac{1}{5\left(2 \cdot \frac{1}{3}+\frac{1}{m}\right)}$ & $\frac{26}{5}, \frac{131}{25}, \ldots$ \\
& & $5+\frac{1}{5\left(2 \cdot \frac{1}{4}-\frac{1}{m}\right)}$ & $\frac{29}{5}, \frac{83}{15}, \ldots$ & $5+\frac{1}{5\left(2 \cdot \frac{1}{4}+\frac{1}{m}\right)}$ & $\frac{79}{15}, \frac{133}{25}, \ldots$ \\
& & $5+\frac{1}{5\left(2 \cdot \frac{1}{5}-\frac{1}{m}\right)}$ & $6, \frac{17}{3}, \ldots$ & $5+\frac{1}{5\left(2 \cdot \frac{1}{5}+\frac{1}{m}\right)}$ & $\frac{16}{3}, \frac{27}{5}, \ldots$
\end{tabular}

where, $\alpha_{i}$ are even numbers and $\sum_{i} \alpha_{i}+1=p$ (with $p$ being a number of loops in $M$-space trajectories which represent $b_{i} b_{i}$ elements). Note that $\frac{1}{m}$ is a fraction of a flux quantum per particle experienced by the closing loop. To obtain correct braid group generators and reasonable wave functions a supplementary requirement is necessary, $\alpha_{i} \bmod \alpha_{j}=0$ if $i \geq j$.

It is worth to mention that also compressible states with particles moving freely like in a Fermi sea without an external magnetic field - can be captured within the topology-based approach to quantum Hall effects. When carriers are building up these (so-called) Hall metal states, then the system is described with multi-looped cyclotron trajectories (representing components of a proper cyclotron subgroup of $\pi_{1}$ ) with the last loop embracing zero flux quanta [16-23]. Lastly, the hierarchy of corresponding filling factors can be established from the generalized hierarchy after introducing of additional mathematical treatment, $m \rightarrow \infty$ $($ or $1 / m \rightarrow 0)$,

$$
\nu=\gamma+\frac{1}{(2 n+1)(p-1)} .
$$

4. Finally, also the pairing of electrons may occur (due to the Fermi sea instability). This action results in a double reduction of particle number and leads to new commensurability conditions,

$$
\left\{\begin{aligned}
\frac{3 h c}{e B}=\frac{3 S}{N_{0}}=\frac{3}{2} \cdot \frac{S}{N-2 N_{0}} \rightarrow \nu & =5 / 2, \\
& \text { up-spin subband } \\
\frac{3 h c}{e B}=\frac{3 S}{N_{0}}=\frac{3}{2} \cdot \frac{S}{N-3 N_{0}} \rightarrow \nu & =7 / 2, \\
& \text { down-spin subband }
\end{aligned}\right.
$$

which can be written in a compressed form (exact for all - except form the LLL - Landau levels),

$$
\begin{aligned}
\frac{(2 n+1) h c}{e B} & =\frac{(2 n+1) S}{N_{0}}=\frac{(2 n+1)}{2} \cdot \frac{S}{N-\gamma N_{0}} \\
& \rightarrow \nu=\gamma+\frac{1}{2} .
\end{aligned}
$$

Note that if one exchanges a real particle number for a diminished number of paired particles $-\frac{(2 n+1)}{2}$. $\frac{S}{\frac{N-\gamma N_{0}}{2}}=(2 n+1) \cdot \frac{S}{N-\gamma N_{0}}-$ the above expression will coincide with one previously presented for the IQHE.

\subsection{Comparison with experiments}

Hall effect investigations - or simply transport measurements - are usually carried out in GaAs/AlGaAs quantum wells symmetrically doped with silicon [6-10,31]. A high electron density and mobility are normally achieved after exposure to red light from a light-emitting diode (LED). Additionally, samples ale cooled down to $T \approx 20-40 \mathrm{mK}$ in various types of refrigerators - dilution, demagnetization or others - equipped with magnets [7,9,31]. Measurements can also be performed in an ultra quiet environment - an environment shielded from electromagnetic noise [6]. What is highly important, all experiments performed so far seem to confirm one, unique observation the FQHE hierarchies of filling factors from higher Landau levels are not counterparts of the Jain hierarchy know from the $L L L[6,8,12]$. This feature was a great surprise impossible to predict within the standard CF model $[4,5]$. In order to deal with this problem scientists presented many possible explanations, mostly concerned on residual interactions of composite fermions (and presence of many 
a)

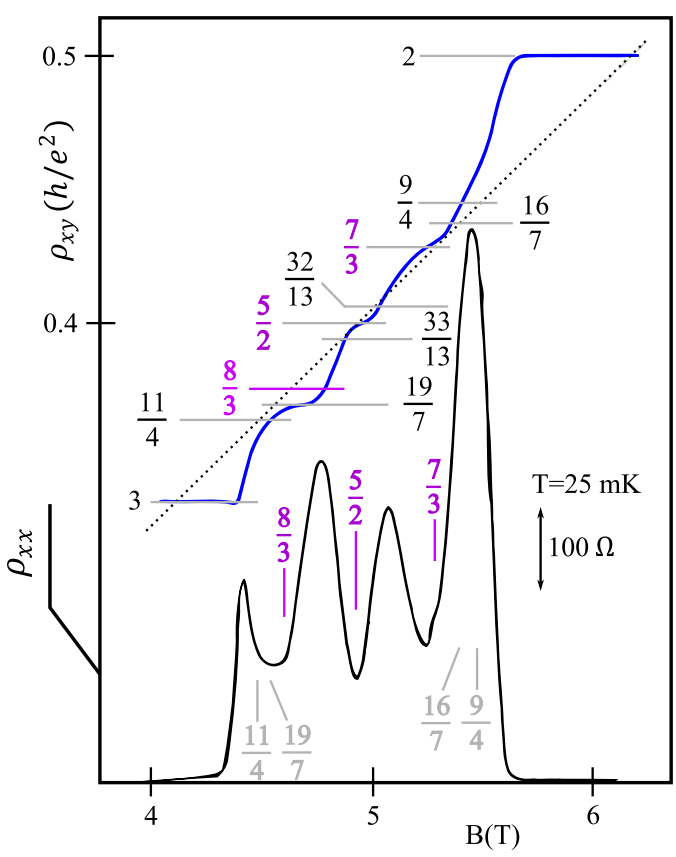

b)

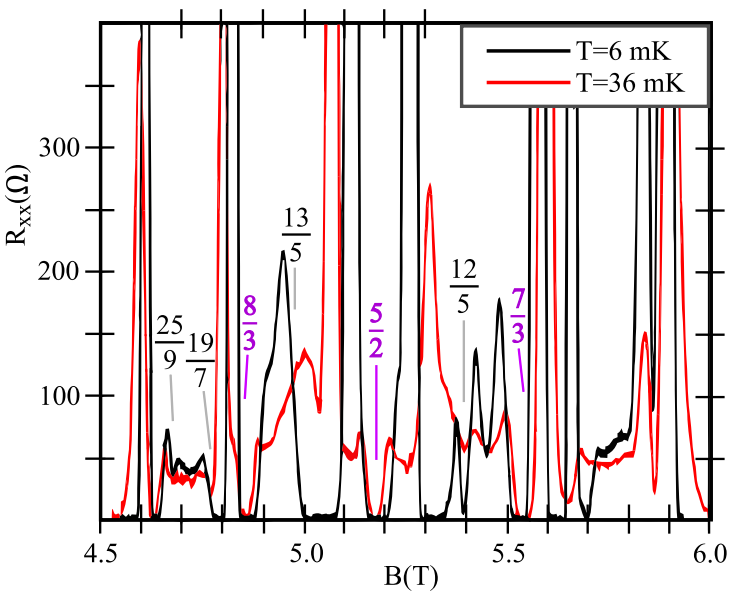

Fig. 2. Transport parameters (longitudinal and transverse resistivities (a) and a longitudinal resistance (b)) as functions of an external magnetic field. The temperatures - at which experiments were carried out - are indicated in the graphs. Additionally, experimentally observed fractions are specified (for filling factors $32 / 13,33 / 13,11 / 4,16 / 7,9 / 4$ only a small quantization accuracy was achieved (a)). The graphs are prepared with the use of references $[7,31]$.

competing ground states, leading to disappearance of the fractional quantum Hall effect). However, it was quickly established - with novel numerical calculations - that complexes (electrons plus flux quanta) cannot generate (in this manner) stable incompressible states observed in transport measurements $[6,32]$. Finally, it was concluded that "the physical mechanism, in which some of the CFs turn into higher-order CFs and condense into new Landau levels to exhibit a quantum Hall effect, does not appear to be relevant for fully polarized electrons" - [32]. Nonetheless, due to the lack of a better explanation (within Jain's model), the idea of residual interactions is still used, while the uneffectiveness of numerical calculations is attributed to the Landau level mixing, the finite-thickness effect and the unknown interaction characteristics not taken into account $[6,7]$.

It is worth to notice that all of these difficulties can be omitted within the topological approach to quantum Hall effects [16-23]. While analyzing considerations included in the cyclotron subgroup model one will arrive with a conclusion that hierarchies in higher Landau levels have to be different - comparing with the Jain's hierarchy in the LLL - due to the rapid enhancement of a particle cyclotron orbit (classical - as a representative of a braid group element) stimulated by the step growth of a kinetic energy, $E_{k}$. The correctness of this model is further confirmed by the fact that all experimentally observed fractions (some of them are presented in Figs. 2 and 3) in the first and second Landau levels can be found in Tables 1 and 2 .

The other interesting feature, noticed in all experimental studies, concerns the robustness of the $\nu=\frac{5}{2}, \frac{7}{3}$ and $\frac{8}{3}$ FQHE states - Figure 3. An unexpectedly high stability of these fractions still lacks of its explanation, despite the intensive research $[7-9,11,31]$, within the Jain's theory of composite fermions $[4,5]$. In reference [8] we find that "the energy gaps of the $\nu=\frac{7}{3}$ and $\frac{8}{3}$ states are disproportionally larger than what may be expected under the standard model of FQHE in the LLL" and "we conclude that the $\frac{7}{3}, \frac{8}{3}$ states are unlikely to be the analogs of the $\frac{1}{3}, \frac{2}{3}$ Laughlin correlated states". Note that this findings perfectly coincide with predictions based on the commensurability conditions $[18,19,22,23]$. All of this states (one paired; $5 / 2$; and two single-loop FQHE; $7 / 3,8 / 3$ ) are generated for the system described with the full braid group (not a cyclotron subgroup) and simple \loopless exchanges (not multi-loop). Thus, they are described by Laughlin correlations with a $p=1$ power in the Jastrow polynomial and are - in a way - more similar to the IQHE than the ordinary FQHE of particles. Lastly, their robustness should exceed one connected with other (ordinary) filling factors, what was precisely confirmed experimentally [8].

Additionally, let us discuss findings presented in reference [12], where transport measurements were conducted with the use of a wide quantum well $(\approx 56 \mathrm{~nm})$. Note that in structures of this type the particle motion (the kinetics) is non-restricted only in a two-dimensional plane of a well, while it is confined $\backslash$ quantized in a perpendicular direction. This confinement leads to the breakdown of the conduction (and valence) band into a set of electric subbands with energies of the edges proportional to $\frac{m^{2}}{a^{2}}$ (where $m$ is natural and symbolizes the subband number, while $a$ stands for the well width). In a typical Hall experiment 

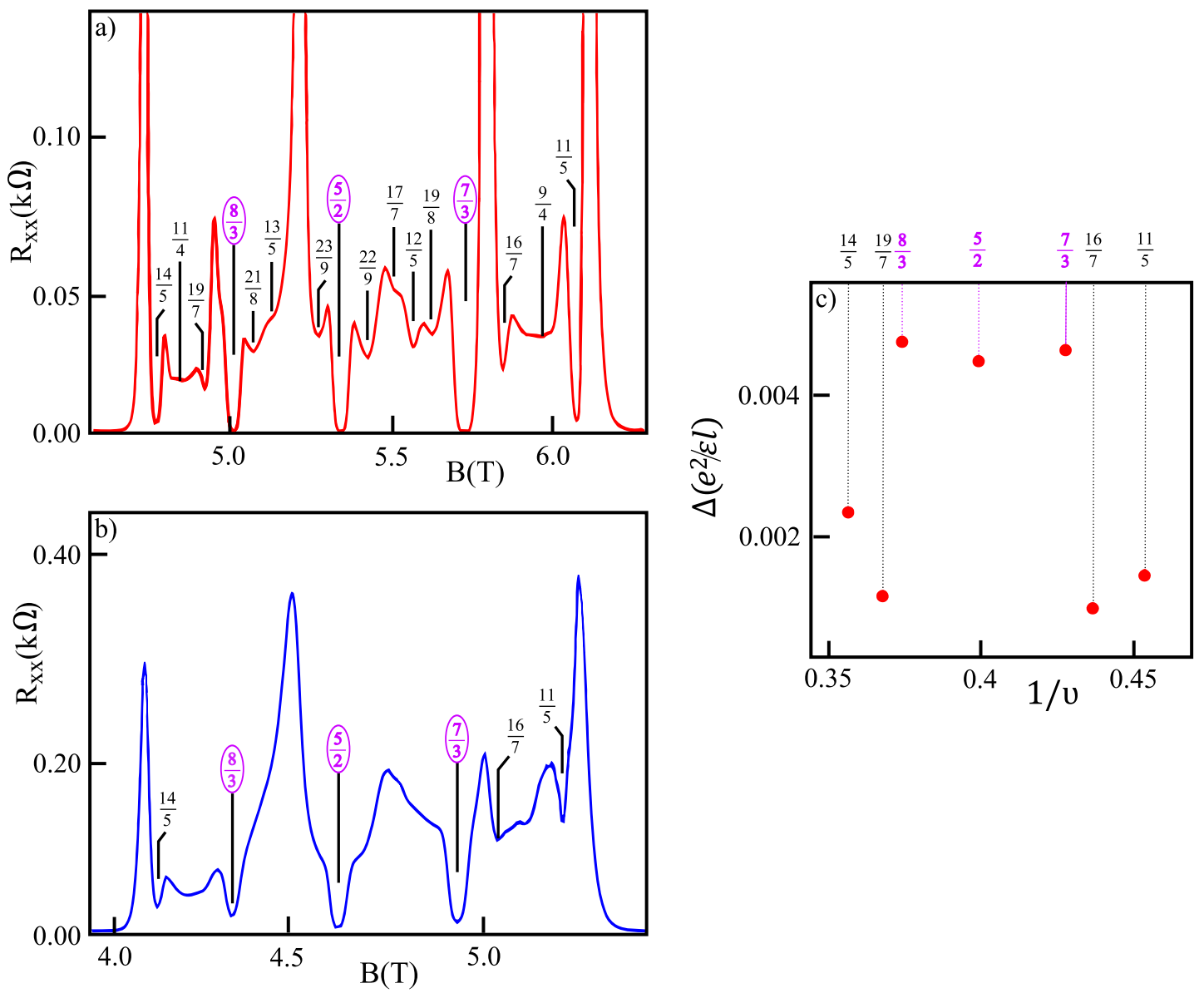

Fig. 3. A longitudinal resistance as a function of an external magnetic field in a high-mobility sample, $\mu=28 \times 10^{6} \mathrm{~cm}^{2} \mathrm{~V}^{-1} \mathrm{~s}^{-1}$ (a) and in a low-mobility sample, $\mu=10.5 \times 10^{6} \mathrm{~cm}^{2} \mathrm{~V}^{-1} \mathrm{~s}^{-1}$ (b). All experimentally observed fractions are indicated. Energy gaps - in the units of the Coulomb energy - for several filling factors in a high-mobility sample (c). The graphs are prepared with the use of reference [8].

very narrow wells are used to assure that electrons occupy only the lowest subband. However, also transport measurements performed in two-subband systems (wide quantum wells, since grater width, $a$, results in smaller separation in energy between the edges) are also enlightening. For example, in reference [12] (Fig. 1) demonstrated that, when the Fermi energy lies in the zeroth Landau level of either of subbands, the even-denominator fractional quantum Hall effect at $\frac{5}{2}$ and $\frac{7}{2}$ fillings is missing. Thus, the appearance of this states requires that the Fermi level is placed within the first Landau level (the case of a singlesubband system). This observation can be easily explained when one notices that the growth of an electron cyclotron orbit (classical, as a representative of a braid from the $\pi_{1}$ ) - forced by the change of a kinetic energy - is necessary to obtain paired states in the multi-particle system (expression (28) and Refs. [18,19,22,23]). Therefore, only when electrons partially fill the first Landau level of an arbitrary electric subband, the $\frac{5}{2}$ and $\frac{7}{2}$ states can be evidenced experimentally.

Finally, let us point out that the location of fractional quantum Hall states close to the subband edges (in higher Landau levels, $n>0$ ) - derived from the cyclotron sub- group model and visible in Tables 1 and 2 - is consistent with experimental observations. Fractions, which are lying in a vicinity of the integer $\nu$ filling, are probably disappearing in its extended dip, what results in further flattening of the main IQHE minimum in a longitudinal resistivity measurement (Fig. 2 in Ref. [11]).

\section{Commensurability conditions in monolayer and bilayer graphene}

In previous sections (and papers [16,17,21-23]) we showed a detailed description of the topology-based model of the FQHE. However, it was derived for typical lowdimensional semiconductors and its use in a monolayer and a bilayer graphene case should be properly explained. To do so, let us examine all of the most important dissimilarities between mentioned materials.

First, it should be noticed that in typical semiconductors the energetic gap between Landau levels is constant and equal to $\Delta E=\hbar \omega_{c}$ (where $\omega_{c}=\frac{e B}{m * c}$ is a cyclotron frequency and $m^{*}$ is an effective mass). While in both graphene structures, due to the gapless spectrum 
and the nonzero Berry phase $(\pi$ or $2 \pi)$, the LLs are not distributed equidistantly [33-36]. However, in the cyclotron subgroups model only the kinetic energy, not the general energy specified by LLs, plays a crucial role due to its proportionality to the size of a particle cyclotron orbit (as a $M$ space mapping of a braid group trajectory from $\Omega$ ). Since the crystal field cannot affect the value of a kinetic energy,

$$
E_{k i n}=\hbar \omega_{c}\left(n+\frac{1}{2}\right)
$$

the cyclotron orbit area is varying with a Landau level index, $n$, in the same manner for graphene, bilayer graphene and other semiconductors. Additionally, the sublevel degeneracy (which can be estimated, for example, as a maximum angular momentum eigenvalue, $m$, in the system of surface $S$ ) is identical in every 2D structure [33,37],

$$
N_{0}=\frac{B S}{h c / e}=\frac{B S}{\phi_{0}}
$$

where, $S$ represent the sample surface (of two sheets in bilayer graphene) and $\phi_{0}=\frac{h c}{e B}$ is a quantum of the magnetic field flux. Despite this, an important disparity between conventional 2DEG and graphene systems, which will be reflected in the topological approach, is related with an unequal number of Landau level subbands (or degrees of freedom like an electronic spin or an isospin) - two in the case of typical systems $(\uparrow, \downarrow)$ and four in the case of graphene structures $\left(K^{\prime} \uparrow, K^{\prime} \downarrow, K \uparrow, K \downarrow\right)$.

There is also another difference in the energy spectra, which leads to a distinct definition of a filling factor $\nu$ in each of cases. In monolayer graphene samples the LLL lies exactly in the Dirac point, while in bilayer samples - not only lowest but also first Landau level [3,33-36,38]. Thus, a half of the $n=0$ level - and $n=1$ for a bilayer - is placed in the valence band and only a remaining half is available for free electrons from the conduction band. Hence, it seems natural to define graphene and bilayer graphene filling factors in terms of an electronic density measured not from the bottom of the LLL, but from the bottom of the lowest spin-valley branch placed in the conduction band $[3,33,36]$. On that basis, a zero filling in graphene $\left(\nu_{g}=0\right)$ corresponds to two completely filled subbands of the LLL, while in bilayer graphene - to four completely filled subbands of the $n=0$ and $n=1$ Landau levels. Finally, the relation between filling factors takes the form of,

$$
\nu=\nu_{g}+2=\nu_{b g}+4
$$

where $\nu_{g}$ is a graphene filling ratio, $\nu_{b g}$ is a bilayer filling ratio and $\nu$ describes all typical semiconductors.

The most important modification of the cyclotron subgroups model of a bilayer graphene is disclosed, when one notices the appearance of an additional surface [19]. In opposition to other structures the bilayer graphene is not strictly two-dimensional, what leads to the significant change in its topological description. The mentioned feature results from the existence of the second sheet, which lies in a close distance and its coupled by a nonzero hopping integral ( $H$ - the Hamiltonian, $\Phi_{A_{2} / B_{1}}$ - the orbital of an $A_{2} / B_{1}$ atom, where $A_{2}$ and $B_{1}$ are from different layers),

$$
\gamma_{1}=\left\langle\Phi_{A_{2}}\left(\vec{r}-\overrightarrow{R_{A_{2}}}\right)|H| \Phi_{B_{1}}\left(\vec{r}-\overrightarrow{R_{B_{1}}}\right)\right\rangle
$$

to the first sheet. Hence, the electrons may freely change positions between the opposite layers (or more accurately - the carrier density is located in both layers). Exchanges of the neighbouring particles (representing braid paths) may be, thus, realized with the participation of electrons from different planes. Let us consider a situation, when the cyclotron orbit, $\frac{(2 n+1) h c}{e B}$, is too short to match the interparticle distance in one layer. In such case, the exchanges of (classical) neighbouring particles are impossible and so is the creation of a collective Hall-like state. The restitution of exchanges and the cyclotron radius enhancement can be achieved by $p$-looped trajectories (constructed with $\frac{p-1}{2}$ looped generators). For a typical semiconductor and monolayer graphene structures the area encircled by these new multi-looped paths (each loop embrace exactly one flux quantum per particle when $\nu=1 / p$ ) equals to [17-19],

$$
S_{c}=p \cdot \frac{(2 n+1) h c}{e B} .
$$

However, the result will be considerably different for bilayer graphene. Since loops of a multi-looped trajectory may be located partly in both $2 \mathrm{D}$ sheets, one needs to take into account the additional surface provided by the second layer. It is expected that the most energetically efficient trajectory corresponds to the situation, when one of loops embrace the surface arising from the supplementary plane, without contributing to the cyclotron area enlargement process (its dimensions are not raised). In this situation, the participation of this one loop (and so the additional surface) in the fulfilment of a commensurability condition must be avoided [19]. Finally, the remaining loops must share the total flux per particle that passes through a single-looped trajectory - that fits to the interparticle distance - independently how they are distributed between two layers. The resulting cyclotron area,

$$
S_{c}=(p-1) \cdot \frac{(2 n+1) h c}{e B} .
$$

Proceeding subsections contain a detailed derivation of a fractional quantum Hall effect hierarchy for both monolayer and bilayer graphene samples. Let us remind that calculations are based on the assumption that a collective Hall-like state can be realized only when mutual exchanges of neighbouring particles (as $M^{N}$ coordinates or arguments of a wave function, $\Psi_{N}$ ) are possible, so a (classical) cyclotron orbit matches the minimal interparticle distance protected by Coulomb repulsion forces. In a correlated multiparticle state, the exchanges - the system braid group generators - are required for the statistics determination. This is due to the fact that multi-particle wave function transformed itself according to a 1DUR of the system $\pi_{1}$. 


\subsection{Filling of the lowest Landau level}

When the first spin-valley branch $0 K^{\prime} \uparrow$ of the LLL is completely filled - a number of particles $N$ coincides with a subband degeneracy $N_{0}$ - a cyclotron orbit fits perfectly to a minimal particle distance. The latter statement is true for both graphene structures. The cyclotron area can be, hence, estimated from the following equation,

$$
N=N_{0}=\frac{B S}{h c / e} \rightarrow \frac{S}{N}=\frac{S}{N_{0}}=\frac{h c}{e B} .
$$

As it was already explained, on the surface defined from (36) falls exactly one flux quantum $\phi_{0}$. Let us once again emphasise that the cyclotron orbit is a classical trajectory, which is understood as a mapping of an element from the system braid group. Though, paths representing braids from the $\pi_{1}$ group are purely classical, it has been proved that their features are reflected in quantummechanical properties of multi-particle systems (1DURs in the Feynman path integral formulation or in transformations of a wave function, $\left.\Psi_{N}\right)$.

After a magnetic field of strength $B$ is raised, the number of particles filling the $0 K^{\prime} \uparrow$ Landau sublevel becomes smaller than its degeneracy $N_{0}$. In this situation, the simple cyclotron path is too small, in comparison with a particle separation, to allow for exchanges in the system. To create a fractional quantum Hall state the enlargement of a cyclotron orbit is necessary and can be provided by the use of multi-looped trajectories (described as a double implementation of multi-looped exchange braids, which are generators of an appropriate cyclotron subgroup). Therefore, the commensurability condition in case of a monolayer graphene takes the form of,

$$
\begin{aligned}
\frac{S}{N} & <\frac{S}{N_{0}} \\
p \frac{h c}{e B} & =p \frac{S}{N_{0}}=\frac{S}{N} \\
\nu_{g} & =\frac{1}{p}=\frac{1}{3}, \frac{1}{5}, \ldots
\end{aligned}
$$

Analogical hierarchy, $\nu_{g}^{h}=1-\frac{1}{p}=\frac{2}{3}, \frac{4}{5}, \ldots$, can be obtained for holes in this spin-valley sublevel. It needs to be clarified that they are not free holes from the valence band - which due to a particle-hole symmetry may be assigned with free electron fractions $\nu_{g}$ taken with a minus sign - but correspond to empty states in the almost filled subband of the conduction band.

The generalized hierarchy (so-called Jain's hierarchy $[4,5])$ can be established if one assumes that $p-1$ loops from the multi-looped trajectory experience exactly one external magnetic field flux quantum per particle, while on the last loop falls only a $\frac{1}{m}$ fraction of $\phi_{0}$ (per particle),

$$
\begin{aligned}
& \nu_{g}=\left((p-1) \pm \frac{1}{m}\right)^{-1}=\frac{m}{m(p-1) \pm 1}, \\
& \nu_{g}^{h}=1-\frac{m}{m(p-1) \pm 1}
\end{aligned}
$$

with cyclotron subgroups generators of the form,

$$
b_{i}^{(p), \pm}=\sigma_{i}^{p-1} \cdot \sigma_{i} \sigma_{i+1} \ldots \sigma_{i+m-1}^{ \pm 1} \ldots \sigma_{i}^{-1} \sigma_{i+1}^{-1} .
$$

If $m$ is an integer, in the zeroth Landau level, then the last loop fits to the minimal separation of every $m$ th particle. However, one can expect some kind of a collective state realization if $m$ is fractional and equals to the inverse number of flux quanta per particle calculated formally for other fillings (from the same or oven other LLs). The latter stands in opposition with the standard composite fermion theory, where $m$ is a number of a completely filled Landau sublevels in a diminished effective magnetic field experienced by CFs, so it must be an integer.

Even denominator filling factors that correspond to the compressible states with particles moving freely like in a Fermi sea without an external magnetic field, can also be established from the cyclotron subgroup picture $[16,17,21-23]$. This fillings are described with multilooped trajectories with one of the loops taking zero flux quanta per particle (specified with a mathematical treatment $m \rightarrow \infty$ ),

$$
\begin{aligned}
& \nu_{g}=((p-1))^{-1}=\frac{1}{(p-1)}=\frac{1}{2}, \frac{1}{4}, \ldots, \\
& \nu_{g}^{h}=1-\frac{1}{(p-1)}=\frac{1}{2}, \frac{3}{4}, \ldots
\end{aligned}
$$

In a bilayer graphene, $p$-looped trajectories are also the recipe for a particle exchanges restitution. However, this material is consisted of two, not one, coupled layers. Thus, one of the loops must utilize the supplementary surface supplied by an additional sheet. This loop does not contribute to a cyclotron orbit enlargement process (effective - the surface of a classical cyclotron orbit remains unchanged and proportional to the magnetic field strength; in $2 \mathrm{D}$ spaces, however, all loops must share the total surface of a trajectory and the flux per particle quantity, $B S / N$, thus each experiences a lower $B$ field) and its dimensions are not raised [19]. Thus, remaining $p-1$ loops must share the same flux as passes through a single-looped orbit, independently how they are distributed between two layers. The resulting commensurability conditions take the form of

$$
\begin{aligned}
& \frac{S}{N_{0}}>\frac{S}{N} \\
& (p-1) \frac{h c}{e B}=(p-1) \frac{S}{N_{0}}=\frac{S}{N} \\
& \nu_{b g}=\frac{1}{p-1}=\frac{1}{2}, \frac{1}{4}, \ldots \\
& \rightarrow \nu_{b g}^{h}=1-\frac{1}{p-1}=\frac{1}{2}, \frac{3}{4}, \ldots
\end{aligned}
$$

Note that the odd denominator fractions seem to form the basic, most robust set in a bilayer graphene samples, at least when a zeroth Landau level subband is filled 
Table 3. Monolayer graphene: filling factors obtained from commensurability conditions for all spin-valley branches of the zeroth Landau level. Odd values of parameter $p$ and integer values of parameter $m$ are considered.

\begin{tabular}{cllll}
\hline LL subb. & IQHE & Hall metal & & FQHE \\
\hline $0 K^{\prime} \uparrow$ & 1 & $\frac{1}{2}, \frac{1}{4}, \ldots$ & $\frac{1}{2-\frac{1}{m}}$ & $1, \frac{2}{3}, \frac{3}{5}, \frac{4}{7}, \frac{5}{9}, \ldots$ \\
& & $\frac{1}{2+\frac{1}{m}}$ & $\frac{1}{3}, \frac{2}{5}, \frac{3}{7}, \frac{4}{9}, \frac{5}{11}, \ldots$ \\
& $\frac{1}{4-\frac{1}{m}}$ & $\frac{2}{7}, \frac{3}{11}, \frac{4}{15}, \ldots$ \\
& $\frac{1}{4+\frac{1}{m}}$ & $\frac{2}{9}, \frac{3}{13}, \frac{4}{17}, \ldots$ \\
\hline $0 K^{\prime} \downarrow$ & 2 & $1+\frac{1}{2-\frac{1}{m}}$ & $2, \frac{5}{3}, \frac{8}{5}, \frac{11}{7}, \frac{14}{9}, \ldots$ \\
& & & $1+\frac{1}{2+\frac{1}{m}}$ & $\frac{4}{3}, \frac{7}{5}, \frac{10}{7}, \frac{13}{9}, \frac{16}{11}, \ldots$ \\
& & $1+\frac{1}{4-\frac{1}{m}}$ & $\frac{4}{3}, \frac{9}{7}, \frac{14}{11}, \ldots$ \\
& & $1+\frac{1}{4+\frac{1}{m}}$ & $\frac{6}{5}, \frac{11}{9}, \frac{16}{13}, \ldots$ \\
\hline
\end{tabular}

Table 4. Bilayer graphene: filling factors obtained from commensurability conditions for all spin-valley branches of the zeroth Landau level. Odd values of parameter $p$ and integer values of parameter $m$ are considered.

\begin{tabular}{cllll}
\hline LL subb. & IQHE & Hall metal & \multicolumn{2}{l}{ FQHE } \\
\hline $0 K^{\prime} \uparrow$ & 1 & $\frac{1}{3}, \frac{1}{5}, \ldots$ & $\frac{1}{1+\frac{1}{m}}$ & $\frac{1}{2}, \frac{2}{3}\left(\frac{1}{3}\right), \frac{3}{4}, \frac{4}{5}, \ldots$ \\
& & $\frac{1}{3-\frac{1}{m}}$ & $\frac{1}{2}, \frac{2}{5}, \frac{3}{8}, \frac{4}{11}, \ldots$ \\
& & $\frac{1}{3+\frac{1}{m}}$ & $\frac{1}{4}, \frac{2}{7}, \frac{3}{10}, \frac{4}{13}, \ldots$ \\
\hline $0 K^{\prime} \downarrow$ & 3 & $\frac{7}{3}, \frac{11}{5}, \ldots$ & $2+\frac{1}{1+\frac{1}{m}}$ & $\frac{5}{2}, \frac{8}{3}, \frac{11}{4}, \frac{14}{5}, \ldots$ \\
& & $2+\frac{1}{3-\frac{1}{m}}$ & $\frac{5}{2}, \frac{12}{5}, \frac{19}{8}, \frac{26}{11}, \ldots$ \\
& & $2+\frac{1}{3+\frac{1}{m}}$ & $\frac{9}{4}, \frac{16}{7}, \frac{23}{10}, \frac{30}{13}, \ldots$ \\
\hline
\end{tabular}

at first. This result perfectly coincides with a recently published transport measurements $[13,14,39]$. Additionally although one of loops is not participating in the cyclotron orbit enlargement process - the total number of loops still equals $p$, resulting in the $b_{i}^{(p)}=\sigma_{i}^{p}$ form of cyclotron subgroup generators and Laughlin correlations with $p$ power in a Jastrow polynomial.

In this picture, a generalized hierarchy - described by trajectories with one loop embracing fractional $\frac{1}{m}$ part of an external magnetic field flux quantum - emerge as,

$$
\begin{aligned}
\nu_{b g} & =\frac{m}{m(p-2) \pm 1}, \\
\nu_{b g}^{h} & =1-\frac{m}{m(p-2) \pm 1} .
\end{aligned}
$$

One should realize that also odd (not only even) denominator fractions, which are observed experimentally [15,39-41], appear in a topology-based model. For example,

$\nu_{b g}=\frac{2}{2 \cdot(3-2)+1}=\frac{2}{3} \quad$ or $\quad \nu_{b g}=\frac{2}{2 \cdot(5-2)-1}=\frac{2}{5}$.
The commensurability conditions (in both graphene structures) remain unchanged in the next subband of the lowest Landau level $0 K^{\prime} \downarrow$, hence the obtained FQHE hierarchy is identical, only shifted by a number of completely filled sublevels. The filling ratios $\nu_{g}$ and $\nu_{b g}$ are presented in a Tables 3 and 4 .

\subsection{Filling of the first Landau level}

In following considerations we assume - what holds for a standard experimental setup [39] - that the sublevels of the $n=0 \mathrm{LL}$ and $n=1 \mathrm{LL}$ are filled alternately in a bilayer graphene case (sequence: $0 K^{\prime} \uparrow, 1 K^{\prime} \uparrow, 0 K^{\prime} \downarrow$, $\left.1 K^{\prime} \downarrow\right)$. In the same time, in a monolayer graphene, states are filled level by level (sequence: $0 K^{\prime} \uparrow, 0 K^{\prime} \downarrow, 1 K^{\prime} \uparrow$, $\left.1 K^{\prime} \downarrow\right)$.

When a magnetic field strength is decreased $B<B_{0}$, particles begin to occupy the first Landau level. During this process electrons located on a partially filled subband ( $\left.1 K^{\prime} \uparrow\right)$ experience the fractional quantum Hall effect, which is accompanied by the IQHE of remaining particles placed on completely filled spin-valley branches lying 
lower on an energy axis. The area of an arbitrary fermion cyclotron orbit (representing element from $\pi_{1}$ ) in the first Landau level is considerably different and equals to,

$$
(2 \cdot 1+1) \frac{h c}{e B}=3 \frac{h c}{e B} .
$$

This sudden growth allows for a realization of new commensurability conditions, all of which are listed below $\left(1 K^{\prime} \uparrow \mathrm{LL}\right)$,

1. The cyclotron orbit may fit perfectly to the interparticle separation, which allows for the exchanges of neighbouring particles (classical - as coordinates of a point in the $M^{N}$ space). In a monolayer graphene case this leads to the expression,

$$
\begin{aligned}
& 3 \frac{h c}{e B}=3 \frac{S}{N_{0}}=\frac{S}{N-2 N_{0}} \\
& \rightarrow \nu_{g}=\frac{7}{3}=2+\frac{1}{3}, \quad \nu_{g}^{h}=3-\frac{1}{3}=\frac{8}{3} .
\end{aligned}
$$

Similarly for a bilayer material,

$$
\begin{aligned}
& 3 \frac{h c}{e B}=3 \frac{S}{N_{0}}=\frac{S}{N-N_{0}} \\
& \rightarrow \nu_{b g}=\frac{4}{3}=1+\frac{1}{3}, \quad \nu_{b g}^{h}=2-\frac{1}{3}=\frac{5}{3} .
\end{aligned}
$$

One should notice that the inequality of obtained fractions, $\nu_{g}$ and $\nu_{b g}$, results solely from a different number of the completely filled spin-valley subbands, $2 N_{0}$ and $N_{0}$. Additionally, although the multi-looped trajectories were not implemented, all filling factors are fractional. Thus, these states correspond to the singlelooped FQHE, rather than the ordinary FQHE [19]. Note that this novel phenomenon is possible only for $n>0$. Even though it is characterized with a fractional quantization of a transverse resistivity (just like the ordinary FQHE), the Laughlin correlations are described with a $p=1$ power in the Jastrow polynomial and a braid group describing the system is generated with loopless generators. Thus, exchanges (as representatives of generators of a system braid group) are single-looped and similar to that in the IQHE. It also seems that this states might be more robust than the ordinary fractional quantum Hall states, what was already confirmed experimentally [42].

2. It is also possible that the surface of a cyclotron orbit (circled in $M$ space and standing for a mapping of a closed trajectory from configuration space) matches the distance of every second,

$$
\begin{aligned}
& 3 \frac{h c}{e B}=3 \frac{S}{N_{0}}=\frac{2 S}{N-2 N_{0}} \rightarrow \nu_{g}=\frac{8}{3}, \quad \nu_{g}^{h}=\frac{7}{3}, \\
& 3 \frac{h c}{e B}=3 \frac{S}{N_{0}}=\frac{2 S}{N-N_{0}} \rightarrow \nu_{b g}=\frac{5}{3}, \quad \nu_{b g}^{h}=\frac{4}{3} \text {. }
\end{aligned}
$$

or every third particle,

$$
\begin{aligned}
& 3 \frac{h c}{e B}=3 \frac{S}{N_{0}}=\frac{3 S}{N-2 N_{0}} \rightarrow \nu_{g}=3, \\
& 3 \frac{h c}{e B}=3 \frac{S}{N_{0}}=\frac{3 S}{N-N_{0}} \rightarrow \nu_{b g}=2 .
\end{aligned}
$$

(Obtained filling factors correspond to the single-loop FQHE and the IQHE, since the cyclotron trajectories are created using simple - without additional loops exchanges.)

3. The most interesting situation occurs when an area of a particle cyclotron orbit is too small to reach electron's nearest neighbour and to provide exchanges in the system (to provide $\sigma_{i}$ generators). However, to form a collective Hall-like state they are necessary and need to be restored with the use of multi-looped trajectories $[16,17,21-23]$. Finally, this commensurability condition leads to the following hierarchy in monolayer graphene,

$$
\begin{aligned}
& \frac{S}{N_{0}}>\frac{S}{N} \\
& p 3 \frac{h c}{e B}=p 3 \frac{S}{N_{0}}=\frac{S}{N-2 N_{0}} \\
& \nu_{g}=\frac{6 p+1}{3 p}=2+\frac{1}{3 p}=\frac{19}{9}, \frac{31}{15}, \ldots \\
& \rightarrow \nu_{g}^{h}=3-\frac{1}{3 p}=\frac{26}{9}, \frac{44}{15}, \ldots
\end{aligned}
$$

and bilayer graphene,

$$
\begin{aligned}
& \frac{S}{N_{0}}>\frac{S}{N} \\
& (p-1) 3 \frac{h c}{e B}=(p-1) 3 \frac{S}{N_{0}}=\frac{S}{N-N_{0}} \\
& \nu_{b g}=\frac{3(p-1)+1}{3(p-1)}=1+\frac{1}{3(p-1)}=\frac{7}{6}, \frac{13}{12}, \ldots \\
& \rightarrow \nu_{g b}^{h}=2-\frac{1}{3(p-1)}=\frac{11}{6}, \frac{23}{12}, \ldots
\end{aligned}
$$

The generalized hierarchies can be established in the same manner as described in the previous subsection,

$$
\begin{aligned}
& \nu_{g}=2+\frac{1}{3\left(p-1 \pm \frac{1}{m}\right)}, \quad \nu_{g}^{h}=3-\frac{1}{3\left(p-1 \pm \frac{1}{m}\right)} \\
& \nu_{b g}=1+\frac{1}{3\left(p-2 \pm \frac{1}{m}\right)}, \quad \nu_{b g}^{h}=3-\frac{1}{3\left(p-2 \pm \frac{1}{m}\right)} .
\end{aligned}
$$

Exemplary fractions are presented in Tables 5 and 6 . Once again we emphasize that other commensurability conditions are worth to consider. Note that a multilooped trajectory may also fit to the separation of every ath particle $(a=1,2,3, \ldots)$. Obtained generalized 
Table 5. Monolayer graphene: filling factors obtained from commensurability conditions for all spin-valley branches of the first Landau level. IQHEp stands for the paired states, while FQHEs - for single-loop states.

\begin{tabular}{|c|c|c|c|c|}
\hline LL subb. & IQHE, IQHEp, FQHEs & Hall metal & & FQHE \\
\hline \multirow[t]{5}{*}{$1 K \uparrow$} & $3, \frac{5}{2}, \frac{7}{3}, \frac{8}{3}$ & $\frac{13}{6}, \frac{25}{12}, \ldots$ & $2+\frac{1}{3\left(2-\frac{1}{m}\right)}$ & $\frac{7}{3}\left(\frac{8}{3}\right), \frac{20}{9}, \frac{11}{5}, \ldots$ \\
\hline & & & $2+\frac{1}{3\left(2+\frac{1}{m}\right)}$ & $\frac{19}{9}, \frac{32}{15}, \frac{15}{7}, \ldots$ \\
\hline & & & $2+\frac{1}{3\left(2 \cdot \frac{1}{2}-\frac{1}{m}\right)}$ & $\frac{8}{3}\left(\frac{7}{3}\right), \frac{22}{9}\left(\frac{23}{9}\right), \frac{12}{5}\left(\frac{13}{5}\right), \ldots$ \\
\hline & & & $2+\frac{1}{3\left(2 \cdot \frac{1}{2}+\frac{1}{m}\right)}$ & $\frac{20}{9}, \frac{34}{15}, \frac{16}{7}, \ldots$ \\
\hline & & & $2+\frac{1}{3\left(2 \cdot \frac{1}{3}-\frac{1}{m}\right)}$ & $3, \frac{8}{3}, \frac{13}{5}\left(\frac{12}{5}\right), \frac{18}{7}\left(\frac{17}{7}\right), \ldots$ \\
\hline \multirow[t]{5}{*}{$1 K \downarrow$} & $4, \frac{7}{2}, \frac{10}{3}, \frac{11}{3}$ & $\frac{19}{6}, \frac{37}{12}, \ldots$ & $3+\frac{1}{3\left(2-\frac{1}{m}\right)}$ & $\frac{10}{3}\left(\frac{11}{3}\right), \frac{29}{9}, \frac{16}{5}, \frac{67}{21}, \ldots$ \\
\hline & & & $3+\frac{1}{3\left(2+\frac{1}{m}\right)}$ & $\frac{28}{9}, \frac{47}{15}, \frac{22}{7}, \ldots$ \\
\hline & & & $3+\frac{1}{3\left(2 \cdot \frac{1}{2}-\frac{1}{m}\right)}$ & $\frac{11}{3}\left(\frac{10}{3}\right), \frac{31}{9}, \frac{17}{5}\left(\frac{18}{5}\right), \ldots$ \\
\hline & & & $3+\frac{1}{3\left(2 \cdot \frac{1}{2}+\frac{1}{m}\right)}$ & $\frac{29}{9}, \frac{23}{7}, \frac{49}{15}, \ldots$ \\
\hline & & & $3+\frac{1}{3\left(2 \cdot \frac{1}{3}-\frac{1}{m}\right)}$ & $4, \frac{11}{3}\left(\frac{10}{3}\right), \frac{18}{5}\left(\frac{17}{5}\right), \frac{25}{7}\left(\frac{24}{7}\right), \ldots$ \\
\hline \multirow[t]{5}{*}{$1 K^{\prime} \uparrow$} & $5, \frac{9}{2}, \frac{13}{3}, \frac{14}{3}$ & $\frac{25}{6}, \frac{49}{12}, \ldots$ & $4+\frac{1}{3\left(2-\frac{1}{m}\right)}$ & $\frac{13}{3}\left(\frac{14}{3}\right), \frac{38}{9}, \frac{21}{5}, \ldots$ \\
\hline & & & $4+\frac{1}{3\left(2+\frac{1}{m}\right)}$ & $\frac{37}{9}, \frac{62}{15}, \frac{29}{7}, \ldots$ \\
\hline & & & $4+\frac{1}{3\left(2 \cdot \frac{1}{2}-\frac{1}{m}\right)}$ & $\frac{14}{3}\left(\frac{13}{3}\right), \frac{40}{9}, \frac{22}{5}\left(\frac{23}{5}\right), \ldots$ \\
\hline & & & $4+\frac{1}{3\left(2 \cdot \frac{1}{2}+\frac{1}{m}\right)}$ & $\frac{38}{9}, \frac{64}{15}, \frac{30}{7}, \ldots$ \\
\hline & & & $4+\frac{1}{3\left(2 \cdot \frac{1}{3}-\frac{1}{m}\right)}$ & $5, \frac{14}{3}\left(\frac{13}{3}\right), \frac{23}{5}\left(\frac{22}{5}\right), \frac{32}{7}\left(\frac{31}{7}\right), \ldots$ \\
\hline \multirow[t]{5}{*}{$1 K^{\prime} \downarrow$} & $5, \frac{11}{2}, \frac{16}{3}, \frac{17}{3}$ & $\frac{31}{6}, \frac{61}{12}, \ldots$ & $5+\frac{1}{3\left(2-\frac{1}{m}\right)}$ & $\frac{16}{3}, \frac{47}{9}, \frac{26}{5}, \ldots$ \\
\hline & & & $5+\frac{1}{3\left(2+\frac{1}{m}\right)}$ & $\frac{46}{9}, \frac{77}{15}, \frac{36}{7}, \ldots$ \\
\hline & & & $5+\frac{1}{3\left(2 \cdot \frac{1}{2}-\frac{1}{m}\right)}$ & $\frac{17}{3}, \frac{49}{9}, \frac{27}{5}, \ldots$ \\
\hline & & & $5+\frac{1}{3\left(2 \cdot \frac{1}{2}+\frac{1}{m}\right)}$ & $\frac{47}{9}, \frac{79}{15}, \ldots$ \\
\hline & & & $5+\frac{1}{3\left(2 \cdot \frac{1}{3}-\frac{1}{m}\right)}$ & $6, \frac{17}{3}, \frac{28}{5}, \frac{39}{7}$, . \\
\hline
\end{tabular}

hierarchies have additional weight factors in a filling factor denominator,

$$
\begin{aligned}
& \nu_{g}=2+\frac{1}{3\left(\frac{p-1}{a} \pm \frac{1}{m}\right)} \\
& \nu_{b g}=1+\frac{1}{3\left(\frac{p-2}{a} \pm \frac{1}{m}\right)}
\end{aligned}
$$

where, $m$ and $a$ are integers and need to satisfy the condition, $m \bmod a=0$.

4. The last condition assumes the possible pairing of electrons (to create bosons), what results in a double reduction of particle number,

$$
\begin{aligned}
& 3 \frac{h c}{e B}=3 \frac{S}{N_{0}}=1.5 \frac{S}{N-2 N_{0}} \rightarrow \nu_{g}=\frac{5}{2} \\
& 3 \frac{h c}{e B}=3 \frac{S}{N_{0}}=1.5 \frac{S}{N-N_{0}} \rightarrow \nu_{b g}=\frac{3}{2} .
\end{aligned}
$$

If one exchanges, in the above equation, the real particle number for a diminished number of paired particles, then the expression will coincide with one previously presented for the IQHE (a phenomenon described with a $p=1$ in Jastrow polynomial).

Similarly as in the lowest Landau level, the presented conditions remain unchanged in the next subbands (three in the case of monolayer and one in the case of bilayer graphene) of the first LL, hence the received quantum Hall hierarchy is identical, only shifted by a number of completely filled sublevels.

It is also very easy to derive commensurability requirements in the higher Landau levels. The only modification appears in the surface of a particle cyclotron orbit (classical), which will fit to separation of every $(2 n+1)$ th particle in the case of a completely filled (arbitrarily chosen) spinvalley branch of the $n$th Landau band. 
Table 6. Bilayer graphene: filling factors obtained from commensurability conditions for all spin-valley branches of the first Landau level. IQHEp stands for the paired states, while FQHEs - for single-loop states.

\begin{tabular}{clll}
\hline LL subb. & IQHE, IQHEp, FQHEs & Hall metal & FQHE (ordinary) \\
\hline $1 K^{\prime} \uparrow$ & $2, \frac{3}{2}, \frac{4}{3}, \frac{5}{3}, \quad \ldots$ & $1+\frac{1}{3\left(1-\frac{1}{m}\right)}$ & $\frac{5}{3}\left(\frac{4}{3}\right), \frac{3}{2}, \frac{13}{9}, \frac{17}{12}, \ldots$ \\
& $1+\frac{1}{3\left(1+\frac{1}{m}\right)}$ & $\frac{7}{6}, \frac{11}{9}, \frac{5}{4}, \frac{19}{15}, \frac{23}{18}, \ldots$ \\
& & $1+\frac{1}{3\left(\frac{1}{2}+\frac{1}{m}\right)}$ & $\frac{4}{3}, \frac{13}{9}, \frac{3}{2}, \frac{23}{15}, \frac{14}{9}, \ldots$ \\
& & $1+\frac{1}{3\left(\frac{1}{3}+\frac{1}{m}\right)}$ & $\frac{3}{2}, \frac{5}{3}, \frac{7}{4}, \frac{9}{5}, \ldots$ \\
\hline $1 K^{\prime} \downarrow$ & & $3+\frac{1}{3\left(1-\frac{1}{m}\right)}$ & $\frac{11}{3}\left(\frac{10}{3}\right), \frac{7}{2}, \frac{31}{9}, \frac{41}{12}, \ldots$ \\
& & $3+\frac{1}{3\left(1+\frac{1}{m}\right)}$ & $\frac{19}{6}, \frac{29}{9}, \frac{13}{4}, \frac{49}{15}, \frac{59}{18}, \ldots$ \\
& & $3+\frac{1}{3\left(\frac{1}{2}+\frac{1}{m}\right)}$ & $\frac{10}{3}, \frac{31}{9}, \frac{7}{2}, \frac{53}{15}, \frac{32}{9}, \ldots$ \\
& & $3+\frac{1}{3\left(\frac{1}{3}+\frac{1}{m}\right)}$ & $\frac{7}{2}, \frac{11}{3}, \frac{15}{4}, \frac{19}{5}, \ldots$ \\
\hline
\end{tabular}

Finally, it should be already clear how additional layers of carbon atoms influence the quantum Hall effect considerations in graphene structures. Any multi-looped trajectory may be located partly in all of the planes, so they must be taken into account while constructing commensurability conditions. As in the case of bilayer graphene, the most energetically efficient arrangement is achieved when exactly one loop embrace the additional surface of an added sheet. Thus, $l$ loops in the case of $(l+1)$ layer graphene are wasted on utilization of supplementary planes, and only remaining $p-l$ can take part in a cyclotron orbit area enhancement. This loops must share the total flux that passes through a single-looped path fitting to the interparticle distance, independently how they are distributed between all of the layers.

\subsection{Comparison with experiments}

Experiments carried out on graphene and its bilayer version are exceptional in many ways. For example, it is possible to modify - with a lateral gate voltage $\left(V_{b g}<\right.$ $10 \mathrm{~V}$ to avoid collapse induced by electrostatic attraction [43]) - the carrier density in a fixed external magnetic field strength, what was not practised in standard 2DEG structures. Additionally, as a result of a small dielectric constant and a great value of energy gap between Landau levels near Dirac points, the anomalous IQHE in monolayer graphene appeared to be extremely robust - the welldefined plateau in transverse resistivity was revealed even in room temperatures [44]. Currently mainly two sample structures - suspended [45] and placed on a boronnitride substrate [46] - are used to develop fractional quantum Hall features in monolayer and bilayer graphene. This dominance can be explained with a great purity of these samples, which allows for the obtainment of ultrahigh carrier mobilities [47]. Furthermore, at least three measuring techniques are worth to consider: the Hall-bar (four-terminal) geometry $[3,39,40,42,48]$, the two-terminal geometry [43,49-51] and the local electronic compressibility measurements $[15,52,53]$.

Firstly, experiments performed on monolayer graphene with the use of four-terminal devices appeared to be remarkably unsuccessful [47], due to small dimensions of samples and the interfering nature of electrodes [50,54]. The invasive voltage-probing contacts (connected directly to the area of a device studied by transport measurements) - placed closely to large potential drop regions and the current-probing terminals - acted like heat sinks, thus allowing electrons with a high value of kinetic energy to leak out through them. This property resulted in the non-effective current annealing [54] - adsorbates were redistributed over a graphene flake, rather than removed from a device body - and in the shorting out of the Hall voltage [50]. The progress was made after the introduction of non-interfering electrodes, connected with a sample by etched constrictions [54]. Finally, many of the FQHE filling factors evidenced experimentally in monolayer graphene were observed in the Hall-bar geometry measurements $[3,42,48]$. Moreover, the experiment carried out in this technique by Amet et al. (Ref. [42]) was the first one to disclose a plethora of collective Hall-like states in the first Landau level (a few single-loop FQHE states had already been presented in the Yacoby group paper, Ref. [53]) - Figure 4. It is also worth to mention that only a separate determination of the longitudinal conductivity, $\sigma_{x x}$, and the transverse one, $\sigma_{x y}$, allows for the observation of fragile (due to small energy gaps) fractional quantum Hall states in bilayer graphene. Since this requirement is unattainable in two-terminal devices - at least not in a straightforward manner (the conductance $G$ actually depends on both $\sigma_{x x}$ and $\sigma_{x y}$ ) - the Hall-bar geometry and the local electronic compressibility measurements dominated the FQHE experiment in bilayer materials $[15,39,40]$. For example, the four-terminal technique made possible observation of a new incompressible state, $\nu=\frac{1}{2}$, which did not occur in standard, two-dimensional heterostructures $[13,14,39]$ - Figure 5. Let us emphasise, 


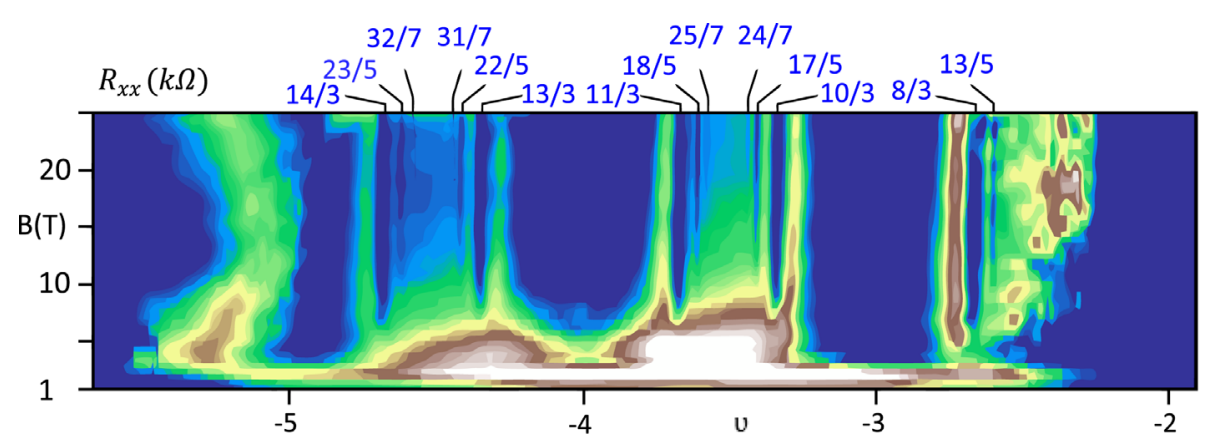

Fig. 4. The longitudinal resistance of a monolayer graphene sample as a function of a magnetic field and a filling factor. $R_{x x} \approx 0.2 \mathrm{k} \Omega$ is colored dark blue, while $R_{x x} \approx 1 \mathrm{k} \Omega$ is colored white. Fractions corresponding to the ordinary FQHE realized in the first Landau level are marked with a blue colour. The diagram is based on a measurement presented in reference [42].
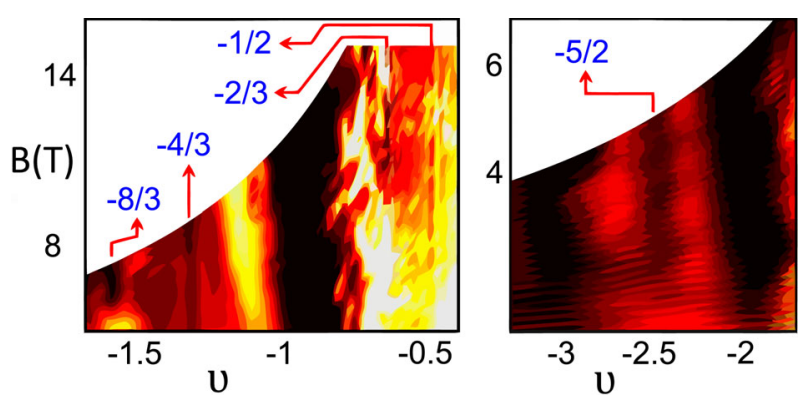

Fig. 5. The longitudinal resistance of a bilayer graphene sample as a function of a magnetic field and a filling factor. $R_{x x} \approx 0 \mathrm{k} \Omega$ is colored dark red, while $R_{x x} \approx 4 \mathrm{k} \Omega$ is colored bright yellow $\backslash$ white. The diagram is based on a measurement presented in reference [39].

that this novel fraction took place of a famous $\nu=\frac{1}{3}-\mathrm{a}$ chief filling factor belonging to the Jain's hierarchy $[4,5]$. Although the appearance of $\nu=1 / 2$ cannot be explained within the composite fermion theory [4,5], the necessity of its occurrence can be easily explained within the cyclotron subgroup model. Though, possible braid subgroups of conventional 2DEG structures (in a magnetic field) are very similar to those of bilayer graphene, in the latter case one loop from a $p$-looped cyclotron trajectory (a representative of a homotopy class belonging to the system $\pi_{1}$ ) needs to utilize an additional surface (layer). As a result, only residual $p-1$ loops share the total, $B S / N$ quantity - [19]Table 4 .

Two-terminal measurements were crucial for the development of the FQHE experiment and a general knowledge about the intrinsic structure of monolayer graphene (especially at the time, when Hall-bar measurements were unsuccessful). It was this geometry that allowed for the first observation of a $\nu=\frac{1}{3}$ incompressible state [43,51] and many others $[49,50]{ }^{-}$in graphene. It is, however, worth to mention that obtaining a detailed description of a collective-Hall like state by using two-terminal devices is not easy nor immediate $[47,50]$. This feature is caused by the fact that a received conductance, $G$, depends not only on a transverse, but also on a longitudinal conductivity. Thus, the estimation of quantitative parameters - like an excitation gap or $\sigma_{x y}$ (even near $\sigma_{x x} \approx 0$ points $)$ - requires an appropriate theoretic approach [50]. The latter can be performed with the use of a so-called Gaussian model, where an effective width-tolength ratio of a graphene flake together with longitudinal conductivity peak positions and widths, for plateau-toplateau transitions between two different collective states $\left(\nu_{1}<\nu_{2}\right)$, are treated as variables [50]. The transverse conductivity, and so the conductance, can be estimated from a semicircle relation, $\sigma_{x x}^{2}=\left(\sigma_{x y}-\nu_{1}\right)\left(\nu_{2}-\sigma_{x y}\right)$. The basic consequence of these difficulties is the inability to obtain well-defined FQHE states in bilayer graphene samples. In one of the papers (presenting two-terminal measurement results) was included an experiment, which intended to demonstrate the existence of a fractional quantum Hall state near $\nu=0.33$ in bilayer samples, but - due to the lack of a strong plateau - it was treated with scepticism and caution [41].

Due to a non-zero disorder level, typical measuring techniques supply only an average (blurry) view of the studied transport parameters [49]. Since monolayer and bilayer graphene samples may be much cleaner on a nanometer scale, thus probing a smaller area may reveal fragile, interaction-induced effects absent in twoand four-terminal experiments (Figs. 6 and 7). Hence, it should not be surprising that local electronic compressibility measurements (performed with single-electron transistors) have recently gained a lot of interest $[15,52,53]$. Additionally, they have been especially successful in locating of collective FQHE states within the LLL of monolayer graphene $[52,53]$ and within higher Landau levels of a bilayer material [15].

Hierarchies obtained from the commensurability conditions for two lowest Landau levels of monolayer and bilayer graphene were presented in Tables 3-6. Note that the appearance of all experimentally observed fractions marked with a pink colour - can be explained within the topological approach to quantum Hall effects. Some of the already measured filling factors can be found in Figures $4-7$. It is worth to emphasise that collective Hall-like states from the first Landau level (with $x=1$ ) seem to gather closely to the subband rims (identified by an integer $\nu$ ). Thus, the FQHE features disappear in highly lengthy dips of a longitudinal resistivity connected with the IQHE. The resultant mixing leads to further extending 
a)

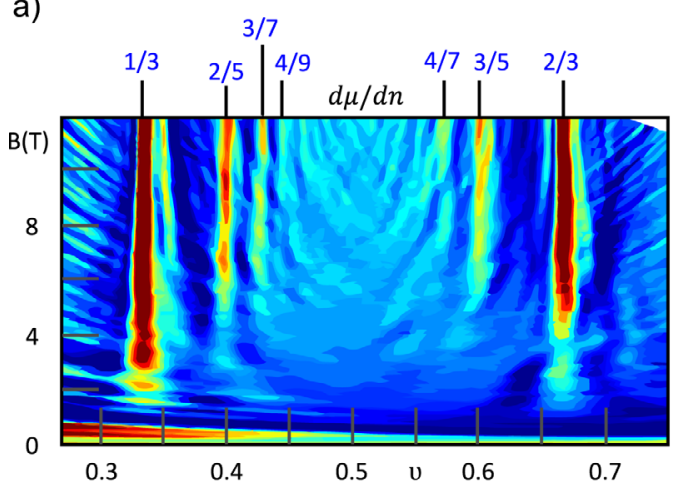

b)

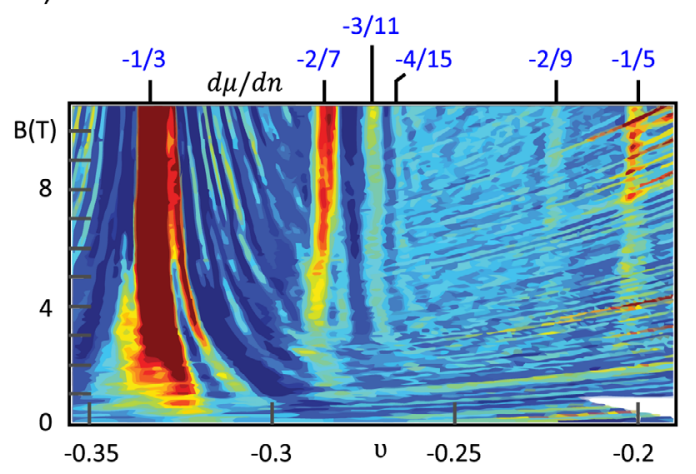

Fig. 6. The inverse compressibility measurements for monolayer graphene samples. $\frac{d \mu}{d n}$ is displayed as a function of a magnetic field strength and a filling factor. In picture (a) $\frac{d \mu}{d n} \approx 4 \times 10^{-17} \mathrm{eV} \mathrm{m}^{2}\left(\approx 2 \times 10^{-16} \mathrm{eV} \mathrm{m}^{2}\right.$ in (b)) is marked with a dark red colour, while $\frac{d \mu}{d n} \approx-2 \times 10^{-17} \mathrm{eV} \mathrm{m}^{2}\left(\approx-1 \times 10^{-16} \mathrm{eV} \mathrm{m}^{2}\right.$ in (b)) is colored with a dark blue colour. The diagram (a) is based on a measurement presented in reference [52] and the diagram (b) - on a measurement presented in reference [53].
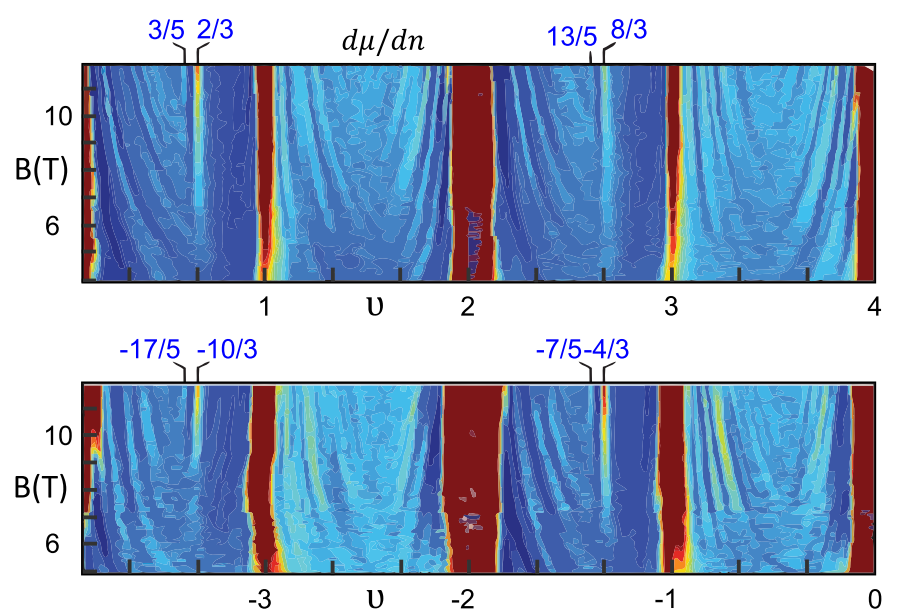

Fig. 7. The inverse compressibility measurements for a bilayer graphene sample. $\frac{d \mu}{d n}$ is displayed as a function of a magnetic field strength and a filling factor. In the picture $\frac{d \mu}{d n} \approx 5 \times$ $10^{-18} \mathrm{eV} \mathrm{m}^{2}$ is marked with a dark red colour, while $\frac{d \mu}{d n} \approx-5 \times$ $10^{-18} \mathrm{eV} \mathrm{m}^{2}$ is colored with a dark blue colour. The diagram is based on a measurement presented in reference [15].

of these already large minimums. These predictions are consistent with experimental observations in monolayer graphene, since a great amount of the theoretically derived Hall-like states stay beyond the resolution ability of measurement techniques. Additionally, the higher the Landau level index is $(n)$, the more and more fractions are pushed towards the subband edges. Note that similar accumulation effect occurs also for higher order commensurability conditions (with $x=2$ or $x=3$ ), but sets of fractions are located closer to the center of a spin branch this is clearly visible in Figure 4.

Finally, we would like to once again point out that in higher Landau levels - states with fractionally quantized $\sigma_{x y}$ may not be responsible for the ordinary FQHE well known from the LLL. If the collective effect is realized in a system described with the full braid group and not a cyclotron subgroup, its nature is assumed to be of the single-loop FQHE type [18,19,22,23]. This novel phenomenon rather resembles the IQHE - due to $p=1$ power in the Jastrow polynomial and loopless exchanges - than the ordinary FQHE. Our conclusions seem to agree with a recently published experiment performed by GoldhaberGordon group (Fig. 1c in Ref. [42]). Dips in a longitudinal resistance associated with filling factors $\nu=\frac{8}{3}, \frac{10}{3}, \frac{11}{3}, \frac{13}{3}$ are developing - in this paper - for magnetic fields as low as $5 \mathrm{~T}$. Thus, the mentioned minima are appearing in fields comparable to those associated with integer $\nu$. It is also worth to emphasize that this happens before the famous $\nu=\frac{1}{3}$ state - and its $\nu=\frac{4}{3}$ twin from the second spin-valley subband of the LLL - emerges. Hence, not all one-third fractions are of the same robustness, what was already predicted by a topology-based approach.

\section{Concluding remarks}

In this paper we presented a detailed description of the topological approach to quantum Hall effects, which is called the cyclotron subgroup model [16-23]. This innovative idea is based on the assumption that a collective Halllike state can be organised only when the particle statistics is determined and, thus, the system is described with the full braid group or its proper subgroup [26]. In twodimensional spaces, the cyclotron motion may confine the variety of accessible multi-particle trajectories (representing braids or, in other words, elements of $\pi_{1}(\Omega)$, where $\Omega$ is a configuration space). For example, when the surface of a cyclotron orbit (classical - as a mapping of a trajectory performed in the configuration space onto the manifold on which particles are placed) is smaller than the minimal area attributed to a single particle $(S / N$, protected with Coulomb repulsion forces), then simple exchanges (or more precisely generators of the full braid group) cannot be realized. For this reason, the external magnetic field appearance may result in the reduction of a collection of permitted classes (containing homotopical trajectories). Finally, only when a subgroup of the full braid group can be created from this reduced set, then the statistic 
can be determined and the Hall effect can be organised. Additionally, the (cyclotron) subgroups of $\pi_{1}$ are generated with multi-loop exchanges of neighbouring particles (classical not quantum - as coordinates of a point in $M^{N}$ space) that for specified filling factors, have sufficient sizes to replace inadmissible simple $\backslash$ loopless exchanges, $\sigma_{i}$. In this paper we also provided a proof that the topological approach leads to surprisingly compact commensurability conditions, which allow for the determination of the FQHE $\nu$ in all Landau levels. Their main meaning can be described in one sentence: the quantum Hall effect can be experienced by electrons $\backslash$ holes in the system if and only if the surface encircled by a single- or multi-looped cyclotron path (as a representative of an element from the system braid group, not a quantum particle trajectory) is equivalent to the area attributed to a particle (or a group of particles).

The LLL hierarchy proposed by Jain $[4,5]$ - which correctness was confirmed experimentally - can be deduced from commensurability requirements. Transport measurements $[6-12,31]$ revealed that pyramids of filling factors belonging to higher Landau levels, $n>0$, are not counterparts of the Jain's hierarchy and are difficult to derive from the standard model of composite fermions (usually an unclear residual interaction of CFs is employed). This feature can be, though, easily explained within the cyclotron subgroup model - the hierarchies need to be different due to the rapid enhancement of a particle cyclotron orbit (stimulated by a step change of the kinetic energy, $\left.E_{k} \propto 2 n+1\right)$. The accuracy of our topological approach is further confirmed by the fact that all experimentally observed fractions are components of the commensurability condition based pyramids of $\nu$ (Tabs. 1, 2 and also 5,6).

Furthermore, the analysis of the cyclotron subgroup model leads to determination of a new multi-particle effect in higher Landau levels, which is called a singleloop FQHE [18,19,22,23]. Even though this novel phenomenon is characterized with a fractional quantization of a transverse resistivity (just like the ordinary FQHE), the Laughlin correlations of some fraction of particles (Halperin class function) are described with a $p=1$ power in the Jastrow polynomial and a braid group of the system is generated with loopless elements. Thus, exchanges are simple and similar to that in the IQHE. It also seems that states correlated with this effect should be more robust than ordinary fractional quantum Hall states, what was already confirmed experimentally in typical semiconductor structures [7-9,11,31] and graphene [42].

In higher Landau levels (when the surface of a cyclotron orbit has sufficient dimensions) also exists the possibility of particle pairing, which results in the appearance of an even-denominator quantum Hall effect near fractions $i+\frac{5}{2}$ (where $i=0,1,2, \ldots$ ). It was recently confirmed in transport measurements performed with the use of a two-subband structures (wide quantum wells) [12] - that this states can indeed appear only when the Fermi energy is placed in the first, not zeroth, Landau level of either of electronic subbands. Otherwise, the deeps in a longitudinal resistance and plateaus in a transverse one are simply not developing.
One of the most important conclusions presented in this paper (and Ref. [19]) refers to the bilayer graphene (and other bilayer materials). As it was already explained, loops belonging to the $p$-loop cyclotron trajectories (representing paths belonging to the homotopy classes of the system braid group) can be located partly in both twodimensional sheets. Thus, while considering commensurability conditions, one needs to take into account an additional surface provided by the upper layer. It is expected that the most favorable configuration is realized, when one of the loops is utilizing (embracing) this supplementary surface. Hence, it cannot take part in a cyclotron orbit enlargement process (effective). Finally, remaining $p-1$ loops must share the total flux per particle - experienced by a single-looped path - independently how they are distributed among two layers. This reasoning allows for an explanation why, in bilayer materials, the famous $\frac{1}{p}=\frac{1}{3}$ is replaced by the novel (previously connected with a Hall metal state rather than a fractional quantum Hall state) $\frac{1}{p-1}=\frac{1}{2}$ filling factor $[13-15]$.

The support from the NCN Project UMO-2011/02/A/ST3/ 00116 is acknowledged.

\section{Author contribution statement}

J.J., P.E. \& L.J. conceived of and designed the study presented in the paper; P.E. drafted the manuscript, and prepared all figures; J.J. \& L.J. helped draft the manuscript. All authors gave final approval for publication.

Open Access This is an open access article distributed under the terms of the Creative Commons Attribution License (http://creativecommons.org/licenses/by/4.0), which permits unrestricted use, distribution, and reproduction in any medium, provided the original work is properly cited.

\section{References}

1. D.C. Tsui, H.L. Stormer, A.C. Gossard, Phys. Rev. Lett. 48, 1559 (1982)

2. R.B. Laughlin, Phys. Rev. B 27, 3383 (1983)

3. C.R. Dean et al., Nat. Phys. 7, 693 (2011)

4. J.K. Jain, Phys. Rev. Lett. 63, 199 (1989)

5. J.K. Jain, Indian J. Phys. 88, 915 (2014)

6. W. Pan et al., Phys. Rev. Lett. 90, 016801 (2003)

7. W. Pan et al., Phys. Rev. B 77, 075307 (2008)

8. H.C. Choi et al., Phys. Rev. B 77, 081301 (2008)

9. C.R. Dean et al., Phys. Rev. Lett. 101, 186806 (2008)

10. R.R. Du et al., Phys. Rev. Lett. 70, 2944 (1993)

11. J.P. Eisenstein et al., Physica E 6, 29 (2000)

12. J. Shabani, Y. Liu, M. Shayegan, Phys. Rev. Lett. 105, 246805 (2010)

13. Y.W. Suen et al., Phys. Rev. Lett. 68, 1379 (1992)

14. J.P. Eisenstein et al., Phys. Rev. Lett. 68, 1383 (1992)

15. A. Kou et al., Science 345, 55 (2014) 
16. J. Jacak, L. Jacak, Europhys. Lett. 92, 60002 (2010)

17. J. Jacak, I. Jóźwiak, L. Jacak, K. Wieczorek, J. Phys: Condens. Matter 22, 355602 (2010)

18. J. Jacak, L. Jacak, Pis'ma v ZhETF 102, 23 (2015)

19. J. Jacak, L. Jacak, Phys. Lett. A 379, 2130 (2015)

20. J. Jacak, I. Jóźwiak, L. Jacak, Phys. Lett. A 374, 346 (2009)

21. J. Jacak, P. Łydżba, L. Jacak, Appl. Math. 6, 345 (2015)

22. J. Jacak, P. Łydżba, L. Jacak, Physica B 475, 122 (2015)

23. P. Łydżba, L. Jacak, J. Jacak, Sci. Rep. 5, 14287 (2015)

24. J.S. Birman, Braids, links, and mappings class groups (Princeton University Press and University of Tokyo Press, 1974)

25. J. Birman, Commun. Pure Appl. Math. 22, 41 (1969)

26. T.D. Imbo, C.S. Imbo, C.S. Sudarshan, Phys. Lett. B 234, 103 (1990)

27. R.P. Feynman, A.R. Hibbs, Quantum Mechanics and Path Integrals (McGraw-Hill Companies, 1966), p. 1

28. R. MacKenzie, arXiv:quant-ph/0004090 (2000)

29. M.G.G. Laidlaw, Phys. Rev. D 3, 1375 (1971)

30. Y. Aharonov, D. Bohm, Phys. Rev. 115, 485 (1959)

31. R. Willett et al., Phys. Rev. Lett. 59, 1776 (1987)

32. S.S. Mandal, J.K. Jain, Phys. Rev. Lett. 66, 155302 (2002)

33. M.O. Goerbig, Rev. Mod. Phys. 83, 1193 (2011)

34. E. McCann, Nanosci. Technol. 57, 237 (2012)
35. V.I. Falko, Philos. Trans. R. Soc. A 366, 205 (2008)

36. K.S. Novoselov et al., Nat. Phys. 2, 17 (2006)

37. K. Klitzing, Rev. Mod. Phys. 58, 519 (1986)

38. Y. Zhang et al., Phys. Rev. Lett. 96, 136806 (2006)

39. D.K. Ki et al., Nano Lett. 14, 2135 (2014)

40. P. Maher et al., Science 345, 62 (2014)

41. W. Bao et al., Phys. Rev. Lett. 105, 246601 (2010)

42. F. Amet et al., Nat. Commun. 6, 5838 (2015)

43. K.I. Bolotin, F. Ghahari, M.D. Shulman, H.L. Stormer, P. Kim, Nature 462, 196 (2009)

44. K.S. Novoselov et al., Science 315, 1379 (2007)

45. J.C. Meyer, Nat. Lett. 446, 60 (2007)

46. C.R. Dean et al., Nat. Nanotechnol. 5, 722 (2010)

47. K.I. Bolotin et al., Solid State Commun. 146, 351 (2008)

48. F. Ghahari, P. Cadden-Zimansky, K. Bolotin, P. Kim, Phys. Rev. Lett. 106, 046801 (2011)

49. D.S. Lee, V. Skakalova, R.T. Weits, K. Klitzing, J.H. Smet, Phys. Rev. Lett. 109, 056602 (2012)

50. I. Skachko et al., Philos. Trans. R. Soc. A 368, 5403 (2010)

51. X. Du, I. Skachko, F. Duerr, A. Luican, E.Y. Andrei, Nature 462, 192 (2009)

52. B.E. Feldman, B. Krauss, J.H. Smet, A. Yacoby, Science 337, 1196 (2012)

53. B.E. Feldman et al., Phys. Rev. Lett. 111, 076802 (2013)

54. D.-K. Ki, A.F. Morpurgo, Nano Lett. 13, 5165 (2013) 